\title{
NEF VECTOR BUNDLES ON A PROJECTIVE SPACE WITH FIRST CHERN CLASS THREE
}

\author{
MASAHIRO OHNO
}

\begin{abstract}
We classify nef vector bundles on a projective space with first Chern class three over an algebraically closed field of characteristic zero; we see, in particular, that these nef vector bundles are globally generated if the second Chern class is less than eight, and that there exist nef but non-globally generated vector bundles with second Chern class eight and nine on a projective plane.
\end{abstract}

\section{INTRODUCTION}

Let $\mathcal{E}$ be a nef vector bundle of rank $r$ on a projective space $\mathbb{P}^{n}$ over an algebraically closed field $K$ of characteristic zero. Let $c_{1}$ be the first Chern class of $\mathcal{E}$. Then $c_{1}$ is non-negative since $\mathcal{E}$ is nef. In [15, Theorem 1], Peternell-Szurek-Wiśniewski classified such $\mathcal{E}$ 's in case $c_{1} \leq 2$, based on the study [18] of Szurek-Wiśniewski. If $c_{1} \leq 2$ and $n \geq 2$, then $\mathbb{P}(\mathcal{E})$ is a Fano manifold, and their proof is based on analysis of contraction morphisms of extremal rays. In [11, §6], a different proof of the classification in case $c_{1} \leq 2$ is given, based on analysis of some twist $\mathcal{E}(d)$ of $\mathcal{E}$ with the full strong exceptional sequence $\mathcal{O}, \mathcal{O}(1), \ldots, \mathcal{O}(n)$ of line bundles.

In this paper we continue our approach to classify nef vector bundles in the next case $c_{1}=3$. Note here that if $c_{1}=3$ then the anti-canonical bundle of $\mathbb{P}(\mathcal{E})$ is nef if $n \geq 2$ and ample if $n \geq 3$. Moreover if $c_{1}=3$ then $0 \leq c_{2} \leq c_{1}^{2}=9$, where $c_{2}$ denotes the second Chern class of $\mathcal{E}$. The main result of this paper is as follows.

Theorem 1. Let $\mathcal{E}$ be a nef vector bundle of rank $r$ on a projective space $\mathbb{P}^{n}$ with $c_{1}=3$. Then $c_{2}$ and $\mathcal{E}$ satisfy one of the following:

(1) $c_{2}=0$ and $\mathcal{E} \cong \mathcal{O}(3) \oplus \mathcal{O}^{\oplus r-1}$;

(2) $c_{2}=2$ and $\mathcal{E} \cong \mathcal{O}(2) \oplus \mathcal{O}(1) \oplus \mathcal{O}^{\oplus r-2}$;

(3) $c_{2}=3$ and $\mathcal{E} \cong \mathcal{O}(1)^{\oplus 3} \oplus \mathcal{O}^{\oplus r-3}$;

(4) $c_{2}=3, n=2$, and $\mathcal{E} \cong T_{\mathbb{P}^{2}} \oplus \mathcal{O}^{\oplus r-2}$;

(In the following, $\mathcal{E}$ fits in one of the following exact sequences.)

(5) $c_{2}=3$ and $0 \rightarrow \mathcal{O}(-1) \rightarrow \mathcal{O}(2) \oplus \mathcal{O}^{\oplus r} \rightarrow \mathcal{E} \rightarrow 0$;

(6) $c_{2}=4$ and $0 \rightarrow \mathcal{O}(-1) \rightarrow \mathcal{O}(1)^{\oplus 2} \oplus \mathcal{O}^{\oplus r-1} \rightarrow \mathcal{E} \rightarrow 0$;

(7) $c_{2}=4, n=3$, and $0 \rightarrow \mathcal{O}(-2) \rightarrow \mathcal{O}(-1)^{\oplus 4} \rightarrow \mathcal{O}(1) \oplus \mathcal{O}^{\oplus r+2} \rightarrow \mathcal{E} \rightarrow 0$;

(8) $c_{2}=4, n=4$, and

$$
0 \rightarrow \mathcal{O}(-3) \rightarrow \mathcal{O}(-2)^{\oplus 5} \rightarrow \mathcal{O}(-1)^{\oplus 10} \rightarrow \mathcal{O}^{\oplus r+6} \rightarrow \mathcal{E} \rightarrow 0
$$

(9) $c_{2}=5$ and $0 \rightarrow \mathcal{O}(-1)^{\oplus 2} \rightarrow \mathcal{O}(1) \oplus \mathcal{O}^{\oplus r+1} \rightarrow \mathcal{E} \rightarrow 0$;

(10) $c_{2}=5, n=3$ or 4 , and $0 \rightarrow \mathcal{O}(-2) \rightarrow \mathcal{O}(-1)^{\oplus 5} \rightarrow \mathcal{O}^{\oplus r+4} \rightarrow \mathcal{E} \rightarrow 0$;

2010 Mathematics Subject Classification. Primary 14F05; Secondary 14J60.

Key words and phrases. nef vector bundles, Fano bundles, spectral sequences.

This work was partially supported by JSPS KAKENHI (C) Grant Number 15K04810. 
(11) $c_{2}=6$ and $0 \rightarrow \mathcal{O}(-1)^{\oplus 3} \rightarrow \mathcal{O}^{\oplus r+3} \rightarrow \mathcal{E} \rightarrow 0$;

(12) $c_{2}=6$ and $0 \rightarrow \mathcal{O}(-2) \rightarrow \mathcal{O}(1) \oplus \mathcal{O}^{\oplus r} \rightarrow \mathcal{E} \rightarrow 0$;

(13) $c_{2}=7$ and $0 \rightarrow \mathcal{O}(-2) \oplus \mathcal{O}(-1) \rightarrow \mathcal{O}^{\oplus r+2} \rightarrow \mathcal{E} \rightarrow 0$;

(14) $c_{2}=8, n=2$, and $0 \rightarrow \mathcal{O}(-2)^{\oplus 2} \rightarrow \mathcal{O}^{\oplus r+1} \oplus \mathcal{O}(-1) \rightarrow \mathcal{E} \rightarrow 0$;

(15) $c_{2}=9$ and $0 \rightarrow \mathcal{O}(-3) \rightarrow \mathcal{O}^{\oplus r+1} \rightarrow \mathcal{E} \rightarrow 0$

(16) $c_{2}=9, n=2$, and $0 \rightarrow \mathcal{O}(-2)^{\oplus 3} \rightarrow \mathcal{O}^{\oplus r} \oplus \mathcal{O}(-1)^{\oplus 3} \rightarrow \mathcal{E} \rightarrow 0$;

(17) $c_{2}=9, n=2$, and

$$
0 \rightarrow \mathcal{O}(-3)^{\oplus r} \rightarrow \mathcal{O}(-2)^{\oplus 3 r+3} \rightarrow \mathcal{O}(-1)^{\oplus 3 r+3} \rightarrow \mathcal{E} \rightarrow 0
$$

(18) $c_{2}=9, n=2$, and $0 \rightarrow \mathcal{O}(-2)^{\oplus 4} \rightarrow \mathcal{O}(1) \oplus \mathcal{O}^{\oplus r-3} \oplus \mathcal{O}(-1)^{\oplus 6} \rightarrow \mathcal{E} \rightarrow 0$;

(19) $c_{2}=9, n \geq 3, c_{3}=27$, and $h^{0}(\mathcal{E}(-1))=1$;

(20) $c_{2}=9, n \geq 4, c_{3}=27, h^{0}(\mathcal{E}(-1))=0$, and $h^{n-3}(\mathcal{E}(2-n))=1$.

Note that every case except the cases (18), (19), and (20) in Theorem 1 is effective: examples of $\mathcal{E}$ in case $(7)$ are $\mathcal{O}(1) \oplus \mathcal{O}^{\oplus r-4} \oplus \Omega_{\mathbb{P}^{3}}(2)$ and $\mathcal{O}(1) \oplus \mathcal{O}^{\oplus r-3} \oplus \mathcal{N}(1)$ where $\mathcal{N}$ is a null correlation bundle on $\mathbb{P}^{3}$ (see Remark 8.1); in case (8), $\mathcal{E}$ is given by a locally free resolution in terms of $\mathcal{O}(-3)$-twist of the full strong exceptional sequence $\mathcal{O}, \mathcal{O}(1), \ldots, \mathcal{O}(4)$ in accordance with [11], but $\mathcal{E}$ is in fact isomorphic to $\Omega_{\mathbb{P}^{4}}(2) \oplus \mathcal{O}^{\oplus r-4}$ (see Remark 8.2); in case (10), if $n=4, \mathcal{E}$ is nothing but an extension of the Tango bundle [19] (see also [14, Chap. I §4.3]) on $\mathbb{P}^{4}$ by a trivial bundle $\mathcal{O}^{\oplus r-3}$, so that $\Omega_{\mathbb{P} 4}^{2}(3)$ is a typical example (see Remark 8.3), and if $n=3$, the restriction of such bundle to a hyperplane $\mathbb{P}^{3}$ is an example; the case (14) comes from Proposition 2 below (see [12] for details); an example in case (16) is given in Example 1; the exact sequence in case (17) in fact derives from Example 3. On the other hand, it is uncertain whether nef vector bundles exist or not in case (18), (19), or (20). Note that neither in case (19) nor in case(20) exist nef vector bundles if they does not exist in case (18), since the restriction to a plane of a nef vector bundle in case (19) or (20) lies in the case (18).

Anghel-Manolache [1] and Sierra-Ugaglia [17] classified globally generated vector bundles on a projective space with first Chern class three. Since global generation implies nefness, Theorem 1 is a generalization of their results. We also note that Langer [9] classified smooth Fano 4-folds with adjunction theoretic scroll structure and $b_{2}=2$; his classification includes that of nef and big rank 2 bundles with $c_{1}=3$ on $\mathbb{P}^{2}$ and $\mathbb{P}^{3}$.

As in the proof in case $c_{1} \leq 2$ in [11], the main feature of our proof of Theorem 1 is an application of the spectral sequence deduced in [13, Theorem 1] from Bondal's theorem [4, Theorem 6.2]. Besides this spectral sequence, some of the key ingredients of our proof are the Riemann-Roch formula, the Kawamata-Viehweg vanishing theorem [7] [22], and the non-negativity of Chern classes of nef vector bundles (see, e.g., [10, Theorem 8.2.1]).

Whereas global generation implies nefness, the converse is not true in general; indeed, the (scheme-theoretic) support of the cokernel of the evaluation map $H^{0}(\mathcal{E}) \otimes \mathcal{O} \rightarrow \mathcal{E}$ in cases (14), (16), and (17) in Theorem 1 is, respectively, a point $w$, a cubic curve $E$, and the whole $\mathbb{P}^{2}$. (If there exists an example in case (18), its corresponding support is also the whole $\mathbb{P}^{2}$. Hence examples in cases (18), (19), or (20) shall be also nef but non-globally generated if they exist.) In fact, the evaluation map in case (14) fits in the exact sequence in the following proposition.

Proposition 2. Given an integer $r \geq 2$ and a point $w$ in a projective plane $\mathbb{P}^{2}$, there exists a vector bundle $\mathcal{E}$ fitting in an exact sequence

$$
0 \rightarrow \mathcal{O}(-3) \rightarrow \mathcal{O}^{\oplus r+1} \rightarrow \mathcal{E} \rightarrow k(w) \rightarrow 0
$$


where $k(w)$ denotes the residue field of the point $w$. Moreover a vector bundle $\mathcal{E}$ fitting in the sequence above is nef but non-globally generated with $c_{1}=3$ and $c_{2}=8$.

In [12, Theorem 1.1], we classified nef vector bundles on a projective space with $c_{1}=3$ and $c_{2}=8$ (based on the previous version of this manuscript): we showed that such bundles exist only on a projective plane and every such bundle derives from the exact sequence given in Proposition 2 and fits in the exact sequence (14) in Theorem 1. Note that the parts of the previous version of the manuscript on which the argument in [12] are relied are kept the same in this major revised manuscript.

This paper is organized as follows. In §2, we recall Bondal's theorem [4, Theorem 6.2] and its related results including the spectral sequence deduced in [13, Theorem 1]. The results in \$2 are fundamental throughout this paper. In \$3, we begin our proof(s) of Theorem 1 (and of Theorem 3 below); based on results in [11, we reduce the problem to the case where $H^{0}(\mathcal{E}(-2))=0$. We also show in $\oint 3$ that this reduction enables us to assume that $3 \leq c_{2}$. Several other formulas - such as the Riemann-Roch formulas - used repeatedly in this paper are also presented in $\$ 3$. In $\$ 4$, we collect some lemmas needed later. In \$5, we give a key lemma, Lemma 5.1, which together with exact sequence (3.16) in $\$ 3.1$ is crucial for the whole proof. In $₫ 6$, we show that nef vector bundles on a projective space with $c_{1}=3$ and $c_{2} \leq 7$ are globally generated (Theorem 3). In \$7, we give a proof of Theorem 1, we omit the proof in case $c_{2} \leq 7$, since we have Theorem 3 and globally generated vector bundles with $c_{1}=3$ are classified in [1] and [17]. Similarly the proof in case $c_{2}=8$ is omitted since it is already given in [12]; thus we only give a proof in case $c_{2}=9$. Note that our presentation of the classification in case $c_{2} \leq 7$ is different from both of [1] and [17]; the situation is the same as in [1] and [17]: the presentations of the classification in [1] and [17] are different because their methods of proofs are different; similarly our presentation differs from those of [1] and [17] because of our new proof. A reader who wonders where the presentation of Theorem 1 in case $c_{2} \leq 7$ comes from may find its proof in the previous version (arXiv 1604.05847 version 4) of the manuscript. In 98 we give several remarks related to the cases (7), (8), and (10) and an example of the case (16) in Theorem 1. We end 98 with a question about the cokernel of the evaluation map $H^{0}(\mathcal{E}) \otimes \mathcal{O} \rightarrow \mathcal{E}$ of a nef vector bundle in case (16) of Theorem 1. In \$9, we give a proof of Proposition 2. Finally note that, since globally generated vector bundles are nef, some properties of globally generated vector bundles also hold more generally for nef vector bundles, but some do not; in $\$ 10$, we give two examples which illustrate that a typical exact sequence for globally generated vector bundles - related to the degeneracy locus of general global sections - does not necessarily exist for nef vector bundles.

1.1. Notation and conventions. Throughout this paper we work over an algebraically closed field $K$ of characteristic zero. Basically we follow the standard notation and terminology in algebraic geometry. For a vector bundle $\mathcal{E}, \mathbb{P}(\mathcal{E})$ denotes Proj $S(\mathcal{E})$, where $S(\mathcal{E})$ denotes the symmetric algebra of $\mathcal{E}$. The tautological line bundle $\mathcal{O}_{\mathbb{P}(\mathcal{E})}(1)$ is also denoted by $H(\mathcal{E})$. We denote by $\mathcal{E}^{\vee}$ the dual of $\mathcal{E}$. For a coherent sheaf $\mathcal{F}$ on a smooth projective variety $X$, we denote by $c_{i}(\mathcal{F})$ the $i$-th Chern class of $\mathcal{F}$. In particular, $c_{i}$ stands for $c_{i}(\mathcal{E})$ of the nef vector bundle $\mathcal{E}$ we are dealing with. We say that a vector bundle is (non-)globally generated if it is (not) generated by global sections. For coherent sheaves $\mathcal{F}$ and $\mathcal{G}$ on $X, h^{q}(\mathcal{F})$ denotes $\operatorname{dim} H^{q}(\mathcal{F})$, and $\operatorname{hom}(\mathcal{F}, \mathcal{G})$ denotes $\operatorname{dim} \operatorname{Hom}(\mathcal{F}, \mathcal{G})$. For any closed subscheme $Z$ in $\mathbb{P}^{n}$, denote by $\mathcal{I}_{Z}$ the ideal sheaf of $Z$ in $\mathbb{P}^{n}$. Finally we refer to [10] for the definition and basic properties of nef vector bundles. 


\section{Preliminaries}

In our proof of Theorem 1, we shall apply repeatedly a spectral sequence deduced in [13, Theorem 1]. So we shall recall this sequence in this section. Note that this sequence is a corollary of Bondal's theorem [4, Theorem 6.2] as can be seen below.

Let $X$ be a smooth projective variety over $K, D^{b}(X)$ the bounded derived category of the abelian category of coherent sheaves on $X$. Assume that there exists a full strong exceptional sequence $G_{0}, \ldots, G_{m}$ in $D^{b}(X)$, and let $G$ be the direct sum $\oplus_{j=0}^{m} G_{j}$ in $D^{b}(X)$.

Recall that if $X=\mathbb{P}^{n}$ then $\mathcal{O}, \mathcal{O}(1), \ldots, \mathcal{O}(n)$ is a full strong exceptional sequence in $D^{b}\left(\mathbb{P}^{n}\right)$ by Beilinson's theorem [3, Theorem].

Let $A$ be the endomorphism ring $\operatorname{End}_{D^{b}(X)}(G)$ of $G$, and let $e_{j}$ be the composite of the projection $G \rightarrow G_{j}$ and the inclusion $G_{j} \rightarrow G$. Then $e_{j} \in A$. Define a right $A$-module $P_{j}$ by $P_{j}=e_{j} A$. The natural isomorphism $A \cong \oplus_{j=0}^{m} P_{j}$ of right $A$-modules implies that $P_{j}$ is a projective right $A$-module. Let $S_{j}$ be the simple right $A$-module such that $S_{j} e_{j} \cong K$ and $S_{j} e_{k} \cong 0$ for all $k \neq j$ and $0 \leq k \leq m$.

Let $D^{b}(\bmod A)$ be the bounded derived category of the abelian category $\bmod A$ of finitely generated right $A$-modules. Bondal's theorem [4, Theorem 6.2] states that $\operatorname{RHom}(G, \bullet)$ : $D^{b}(X) \rightarrow D^{b}(\bmod A)$ is an exact equivalence. Since $\bullet \otimes^{\mathrm{L}} G: D^{b}(\bmod A) \rightarrow D^{b}(X)$ is a quasi-inverse of $\operatorname{RHom}(G, \bullet)$, we have a natural isomorphism

$$
\operatorname{RHom}(G, \bullet) \otimes^{\mathrm{L}} G \cong
$$

of functors on $D^{b}(X)$. If we write down this isomorphism for a coherent sheaf $F$ on $X$ in terms of a spectral sequence, we obtain the following spectral sequence ([13, Theorem 1])

$$
E_{2}^{p, q}=\mathcal{T}_{o r}^{A}{ }_{-p}\left(\operatorname{Ext}^{q}(G, F), G\right) \Rightarrow E^{p+q}=\left\{\begin{array}{lll}
F & \text { if } & p+q=0 \\
0 & \text { if } & p+q \neq 0
\end{array}\right.
$$

We call this sequence the Bondal spectral sequence. In practice, in order to apply the Bondal spectral sequence (2.1), we need to compute $E_{2}^{p, q}$. One way to compute $E_{2}^{p, q}$ is by definition: $E_{2}^{p, q}=\mathcal{T}_{o r}{ }_{-p}^{A}\left(\operatorname{Ext}^{q}(G, F), G\right)$, i.e., through a projective resolution of the right $A$-module $\operatorname{Ext}^{q}(G, F)$. Recall here (see [11, Lemma 2.1] for a proof) that a finitely generated right $A$-module $\operatorname{Ext}^{q}(G, F)$ has a projective resolution of the form

$$
0 \rightarrow P_{0}^{\oplus e_{m, 0}} \rightarrow \cdots \rightarrow \bigoplus_{j=0}^{m-l} P_{j}^{\oplus e_{l, j}} \rightarrow \cdots \rightarrow \bigoplus_{j=0}^{m} P_{j}^{\oplus e_{0, j}} \rightarrow \operatorname{Ext}^{q}(G, F) \rightarrow 0
$$

where $e_{0, j}=\operatorname{dim} \operatorname{Ext}^{q}\left(G_{j}, F\right)$ for $0 \leq j \leq m$ and $e_{l, j}$ is determined inductively for $l \geq 1$ and $j \leq m-l$ by the following formula:

$$
e_{l, j}=\sum_{j<k} e_{l-1, k} \operatorname{hom}\left(G_{j}, G_{k}\right)
$$

We shall freely use the following isomorphism:

$$
P_{j} \otimes_{A}^{\mathrm{L}} G=P_{j} \otimes_{A} G \cong G_{j} .
$$

This isomorphism together with (2.2) implies that $E_{2}^{p, q}$ is the $(-p)$-th homology of the following complex

$$
0 \rightarrow G_{0}^{\oplus e_{m, 0}} \rightarrow \cdots \rightarrow \bigoplus_{j=0}^{m-l} G_{j}^{\oplus e_{l, j}} \rightarrow \cdots \rightarrow \bigoplus_{j=0}^{m} G_{j}^{\oplus e_{0, j}} \rightarrow 0 .
$$


Throughout this paper, we set $X=\mathbb{P}^{n}, m=n$, and $G_{j}=\mathcal{O}(j)$ for $0 \leq j \leq n$, and we fix the notation $G_{j}, G, A, P_{j}$, and $S_{j}$ as above.

A typical projective resolution of the form (2.2) in this paper is in the case where $q=0$ and $F=\mathcal{E}(d)$ for some non-negative integer $d$. For example, if $\operatorname{hom}(\mathcal{O}(2), \mathcal{E}(d))=0$, we frequently and sometimes implicitly consider a projective resolution of the form

$$
0 \rightarrow P_{0}^{\oplus(n+1) e_{0,1}} \rightarrow P_{1}^{\oplus e_{0,1}} \oplus P_{0}^{\oplus e_{0,0}} \rightarrow \operatorname{Hom}(G, \mathcal{E}(d)) \rightarrow 0
$$

where $e_{0,0}=\operatorname{hom}(\mathcal{O}, \mathcal{E}(d))$ and $e_{0,1}=\operatorname{hom}(\mathcal{O}(1), \mathcal{E}(d))$.

Finally note that the Bott formula [14, p. 8] implies $\operatorname{RHom}\left(G, \Omega_{\mathbb{P}^{n}}^{j}(j)\right) \cong S_{j}[-j]$ for $0 \leq j \leq n$. Hence we have isomorphisms

$$
S_{j} \otimes_{A}^{\mathrm{L}} G \cong \Omega_{\mathbb{P}^{n}}^{j}(j)[j] .
$$

for $0 \leq j \leq n$. In particular,

$$
S_{n} \otimes^{\mathrm{L}}{ }_{A} G \cong \mathcal{O}(-1)[n] .
$$

Note that this gives another way to compute $E_{2}^{p, q}=\mathcal{H}^{p}\left(\operatorname{Ext}^{q}(G, \mathcal{E}(d)) \otimes^{\mathrm{L}}{ }_{A} G\right)$ : through a filtration of $\operatorname{Ext}^{q}(G, \mathcal{E}(d))$ with subquotients the direct sums of the simple modules $S_{j}$. For example, if $\operatorname{Ext}^{q}(G, \mathcal{E}(d)) \cong S_{j}$ then $E_{2}^{p, q}=\mathcal{H}^{p}\left(\Omega_{\mathbb{P} n}^{j}(j)[j]\right)$, and thus $E_{2}^{p, q}=0$ if $p \neq-j$ and $E_{2}^{-j, q}=\Omega_{\mathbb{P}^{n}}^{j}(j)$. In particular, if $\operatorname{Ext}^{q}(G, \mathcal{E}(d)) \cong S_{n}$ then $E_{2}^{-n, q}=\mathcal{O}(-1)$ and $E_{2}^{p, q}=0$ if $p \neq-n$. We shall also use these formulas frequently.

\section{Set-up And formulas for the proofs of Theorems 1 and 3}

In this section, we give some preparatory parts of our proofs of Theorems 1 and 3 , and we also collect several formulas needed later.

Let $\mathcal{E}$ be a nef vector bundle of rank $r$ on a projective space $\mathbb{P}^{n}$ with $c_{1}=3$. If $\operatorname{Hom}(\mathcal{O}(3), \mathcal{E}) \neq 0$, then $\mathcal{E} \cong \mathcal{O}(3) \oplus \mathcal{O}^{\oplus r-1}$ by [11, Proposition 5.2 and Remark 5.3]. This is the case (1) of Theorem 11. Assume that $\operatorname{Hom}(\mathcal{O}(3), \mathcal{E})=0$. Then $r \geq 2$. If $\operatorname{Hom}(\mathcal{O}(2), \mathcal{E}) \neq 0$, then it follows from [11, Theorem 6.4] that $\mathcal{E}$ is in the case (2) or (5) of Theorem 1 .

In the rest of our proof, we always assume that

$$
H^{0}(\mathcal{E}(-2))=\operatorname{Hom}(\mathcal{O}(2), \mathcal{E})=0 .
$$

Recall that the Kodaira vanishing theorem implies that

$$
H^{q}\left(\left.\mathcal{E}\right|_{L^{l}}(3-k)\right)=0
$$

for all $q>0$, all $l$-dimensional linear subspaces $L^{l} \subseteq \mathbb{P}^{n}$, and all $k \leq l$, since $\mathcal{E}$ is a nef vector bundle with $c_{1}=3$ (see [11, Lemma $4.1(1)$ ] for a proof). If $H\left(\left.\mathcal{E}\right|_{L^{l}}\right)$ is big in addition, then the Kawamata-Viehweg vanishing theorem implies that

$$
H^{q}\left(\left.\mathcal{E}\right|_{L^{l}}(2-k)\right)=0
$$

for all $q>0$ and all $k \leq l$ (see [11, Lemma 4.1 (2)] for a proof).

Since $\mathcal{E}$ is nef, $H^{q}\left(\left.\mathcal{E}\right|_{L}(1)\right)=0$ for all $q>0$ and all lines $L$ in $\mathbb{P}^{n}$. Together with (3.2), this implies that $H^{2}\left(\left.\mathcal{E}\right|_{L^{2}}\right)=0$ for any plane $L^{2} \subseteq \mathbb{P}^{n}$. This vanishing $H^{2}\left(\left.\mathcal{E}\right|_{L^{2}}\right)=0$ then implies that $H^{2}\left(\left.\mathcal{E}\right|_{L^{2}}(-1)\right)=0$ since $H^{q}\left(\left.\mathcal{E}\right|_{L}\right)=0$ for all $q>0$. Moreover we have $H^{2}\left(\left.\mathcal{E}\right|_{L^{2}}(-2)\right)=0$ since $H^{q}\left(\left.\mathcal{E}\right|_{L}(-1)\right)=0$ for all $q>0$. Summing up, we have

$$
H^{2}\left(\left.\mathcal{E}\right|_{L^{2}}(-k)\right)=0
$$

for all $k \leq 2$ and any plane $L^{2}$ in $\mathbb{P}^{n}$. 
If $n=2$, the Riemann-Roch formula for a twisted vector bundle $\mathcal{E}(t)$ is

$$
\chi(\mathcal{E}(t))=\frac{1}{2}(r t+6)(t+3)+r-c_{2} .
$$

Recall that this formula is for $c_{1}=c_{1}(\mathcal{E})=3$. It follows from this formula that $\chi(\mathcal{E}(-2))=$ $3-c_{2}$. For arbitrary $n \geq 2$, the vanishing (3.4) then implies that

$$
h^{0}\left(\left.\mathcal{E}\right|_{L^{2}}(-2)\right)-h^{1}\left(\left.\mathcal{E}\right|_{L^{2}}(-2)\right)=3-c_{2}\left(\left.\mathcal{E}\right|_{L^{2}}\right)
$$

for any plane $L^{2}$ in $\mathbb{P}^{n}$.

We claim here that

$$
c_{2} \geq 3
$$

on $\mathbb{P}^{n}$. Suppose, to the contrary, that $c_{2} \leq 2$. Then $\chi\left(\left.\mathcal{E}\right|_{L^{2}}(-2)\right)=3-c_{2}\left(\left.\mathcal{E}\right|_{L^{2}}\right) \geq 1$ since $c_{2}=c_{2}\left(\left.\mathcal{E}\right|_{L^{2}}\right)$. Hence we obtain $h^{0}\left(\left.\mathcal{E}\right|_{L^{2}}(-2)\right) \neq 0$. As we have seen, this implies that $\left.\mathcal{E}\right|_{L^{2}}$ lies either in the case (1), (2), or (5) of Theorem 1, if $\left.\mathcal{E}\right|_{L^{2}}$ lies in the case (5) then $c_{2}\left(\left.\mathcal{E}\right|_{L^{2}}\right)=3$, which contradicts that $c_{2} \leq 2$. Thus $\left.\mathcal{E}\right|_{L^{2}}$ actually lies either in the case (1) or (2) of Theorem 1. In particular it splits into a direct sum of line bundles. Hence $\mathcal{E}$ also splits by the splitting criterion [14, Theorem 2.3.2] of Horrocks. Since $h^{0}\left(\left.\mathcal{E}\right|_{L^{2}}(-2)\right) \neq 0$, this implies that $h^{0}(\mathcal{E}(-2)) \neq 0$, which contradicts the assumption (3.1). Hence the claim follows.

Since $\left.\mathcal{E}\right|_{L^{2}}$ is nef, we have an inequality $0 \leq H\left(\left.\mathcal{E}\right|_{L^{2}}\right)^{r+1}$. Since $H\left(\left.\mathcal{E}\right|_{L^{2}}\right)^{r+1}$ is equal to $c_{1}\left(\left.\mathcal{E}\right|_{L^{2}}\right)^{2}-c_{2}\left(\left.\mathcal{E}\right|_{L^{2}}\right)$ (see [6, $\S 3.1$ and $\left.\left.\S 3.2\right]\right)$, we obtain $c_{2}\left(\left.\mathcal{E}\right|_{L^{2}}\right) \leq 9$. Hence we have

$$
c_{2} \leq 9 \text {. }
$$

Recall here the well-known non-negativity (see, e.g., [10, Theorem 8.2.1]) of the top Chern class of a nef vector bundle $\mathcal{E}$ that

$$
c_{n} \geq 0 \text {. }
$$

We shall divide the proof of Theorem 1 according to the value of $c_{2} \geq 3$.

Note that $H\left(\left.\mathcal{E}\right|_{L^{2}}\right)$ is nef and big if $c_{2}<9$. The vanishing (3.3) then implies that

$$
H^{q}\left(\left.\mathcal{E}\right|_{L^{2}}\right)=0
$$

for any $q>0$ and any plane $L^{2}$ in $\mathbb{P}^{n}$. Together with the vanishing (3.2), this implies that

$$
H^{q}\left(\left.\mathcal{E}\right|_{L^{3}}(-1)\right)=0
$$

for any $q \geq 2$ and any three-dimensional linear subspace $L^{3} \subset \mathbb{P}^{n}$.

3.1. Set-up for the two-dimensional case with $h^{1}(\mathcal{E})=0$. Note that the vanishing $h^{1}(\mathcal{E})=0$ holds if $c_{2}<9$ by (3.8). It follows from (3.1) and (3.6) that

$$
h^{1}(\mathcal{E}(-2))=c_{2}-3 .
$$

The Riemann-Roch formula (3.5) shows that $\chi(\mathcal{E}(-1))=6-c_{2}$ and $\chi(\mathcal{E})=9+r-c_{2}$. Since we have the vanishings (3.4) and $h^{1}(\mathcal{E})=0$, these formulas imply

$$
\begin{gathered}
h^{0}(\mathcal{E}(-1))-h^{1}(\mathcal{E}(-1))=6-c_{2}, \\
h^{0}(\mathcal{E})=9+r-c_{2} .
\end{gathered}
$$

Since we have an exact sequence $\left.0 \rightarrow \mathcal{E}(-2) \rightarrow \mathcal{E}(-1) \rightarrow \mathcal{E}\right|_{L}(-1) \rightarrow 0$, by taking cohomology, we see that

$$
h^{1}(\mathcal{E}(-2)) \geq h^{1}(\mathcal{E}(-1)) .
$$


Now we apply to $\mathcal{E}$ the Bondal spectral sequence (2.1). It is clear that $E_{2}^{p, q}=0$ if $q<0$ or $p>0$. The vanishing (3.4) shows that $E_{2}^{p, q}=0$ if $q \geq 2$. Since $H^{1}(\mathcal{E})=0$ by assumption, the right $A$-module $\operatorname{Ext}^{1}(G, \mathcal{E})$ fits in an exact sequence

$$
0 \rightarrow S_{1}^{\oplus h^{1}(\mathcal{E}(-1))} \rightarrow \operatorname{Ext}^{1}(G, \mathcal{E}) \rightarrow S_{2}^{\oplus h^{1}(\mathcal{E}(-2))} \rightarrow 0 .
$$

Since $S_{1} \otimes^{\mathrm{L}}{ }_{A} G \cong \Omega_{\mathbb{P}^{2}}(1)[1]$ and $S_{2} \otimes^{\mathrm{L}}{ }_{A} G \cong \mathcal{O}(-1)[2]$ by (2.4), the sequence above induces the following distinguished triangle in $D^{b}(X)$ :

$$
\mathcal{O}(-1)^{\oplus h^{1}(\mathcal{E}(-2))}[1] \rightarrow \Omega_{\mathbb{P}^{2}}(1)^{\oplus h^{1}(\mathcal{E}(-1))}[1] \rightarrow \operatorname{Ext}^{1}(G, \mathcal{E}) \otimes^{\mathrm{L}}{ }_{A} G \rightarrow
$$

Since $E_{2}^{p, 1}=\mathcal{H}^{p}\left(\operatorname{Ext}^{1}(G, \mathcal{E}) \otimes{ }^{\mathrm{L}}{ }_{A} G\right)$, the triangle above shows that $E_{2}^{p, 1}=0$ unless $p=-2$ or -1 and that $E_{2}^{-2,1}$ and $E_{2}^{-1,1}$ fit in the following exact sequence of coherent sheaves:

$$
0 \rightarrow E_{2}^{-2,1} \rightarrow \mathcal{O}(-1)^{\oplus h^{1}(\mathcal{E}(-2))} \stackrel{\mu}{\rightarrow} \Omega_{\mathbb{P}^{2}}(1)^{\oplus h^{1}(\mathcal{E}(-1))} \rightarrow E_{2}^{-1,1} \rightarrow 0 .
$$

It follows from (3.1) that the right $A$-module $\operatorname{Hom}(G, \mathcal{E})$ has, as in (2.3), a projective resolution of the form

$$
0 \rightarrow P_{0}^{\oplus 3 e_{0,1}} \rightarrow P_{1}^{\oplus e_{0,1}} \oplus P_{0}^{\oplus e_{0,0}} \rightarrow \operatorname{Hom}(G, \mathcal{E}) \rightarrow 0
$$

where $e_{0,0}=h^{0}(\mathcal{E})$ and $e_{0,1}=h^{0}(\mathcal{E}(-1))$. In particular, we see that $E_{2}^{p, 0}=0$ if $p<-1$. We have the following exact sequence

$$
0 \rightarrow E_{2}^{-2,1} \rightarrow E_{2}^{0,0} \rightarrow E_{3}^{0,0} \rightarrow 0
$$

Now the Bondal spectral sequence implies that $E_{2}^{-1,0}=0$ and that $\mathcal{E}$ fits in an exact sequence

$$
0 \rightarrow E_{3}^{0,0} \rightarrow \mathcal{E} \rightarrow E_{2}^{-1,1} \rightarrow 0
$$

Since $E_{2}^{-1,0}=0, E_{2}^{0,0}$ fits in an exact sequence

$$
0 \rightarrow \mathcal{O}^{\oplus 3 e_{0,1}} \rightarrow \mathcal{O}(1)^{\oplus e_{0,1}} \oplus \mathcal{O}^{\oplus e_{0,0}} \rightarrow E_{2}^{0,0} \rightarrow 0 .
$$

The following lemma shall be applied repeatedly throughout this paper.

Lemma 3.1. For any finite morphism $C \rightarrow \mathbb{P}^{2}$ from a smooth projective curve $C$, the pullback $\left.E_{2}^{-1,1}\right|_{C}$ of the sheaf $E_{2}^{-1,1}$ can not admit a line bundle of negative degree as a quotient. In particular, $E_{2}^{-1,1}$ can not admit the following sheaves as a quotient:

(1) $\Omega_{\mathbb{P}^{2}}(1)$;

(2) $\mathcal{I}_{p}$, where $p$ is a point;

(3) $\mathcal{I}_{Z}(1)$, where $Z$ is a 0 -dimensional closed subscheme of length $Z \geq 2$;

(4) $\mathcal{I}_{Z}(2)$, where $Z$ is a 0 -dimensional closed subscheme of length $Z \geq 5$;

(5) $\mathcal{O}_{L}(-p)$, where $L$ is a line passing through a point $p$;

(6) $\mathcal{O}_{C}(-p)$, where $C$ is a conic passing through a point $p$.

Proof. The first statement follows from the sequence (3.16) since $\mathcal{E}$ is nef. The second statement is almost obvious from the first, so that we only give a proof in case (4). If there exists a line $L$ such that length $Z \cap L \geq 3$, then the double twist $\mathcal{I}_{Z} \cdot \mathcal{O}_{L}(2)$ of the inverse image ideal sheaf $\mathcal{I}_{Z} \cdot \mathcal{O}_{L}$ has negative degree, which contradicts the first statement. If length $Z \cap L \leq 2$ for any line $L$, then Lemma 4.4 below shows that there exists a smooth conic $C$ such that length $Z \cap C \geq 5$. Hence $\mathcal{I}_{Z} \cdot \mathcal{O}_{C}(2)$ has negative degree, which contradicts the first statement again. Therefore $E_{2}^{-1,1}$ can not admit $\mathcal{I}_{Z}(2)$ as a quotient if length $Z \geq 5$. 
Lemma 3.1 and the exact sequence (3.14) indicate that there are some relations between $h^{1}(\mathcal{E}(-2))$ and $h^{1}(\mathcal{E}(-1))$; we shall explore these relations in Lemma 5.1,

3.2. Set-up for the three-dimensional case. The Riemann-Roch formula for a twisted vector bundle $\mathcal{E}(t)$ on $\mathbb{P}^{3}$ is

$$
\chi(\mathcal{E}(t))=\frac{1}{2}\left\{9-3 c_{2}+c_{3}+\left(9-2 c_{2}\right)(t+2)+\left(3 t^{2}+12 t+11\right)\right\}+\frac{r}{6}(t+3)(t+2)(t+1) .
$$

Recall that this formula is for $c_{1}=c_{1}(\mathcal{E})=3$. By this formula, we have

$$
\begin{gathered}
\chi(\mathcal{E}(-3))=1-\frac{1}{2}\left(c_{2}-c_{3}\right), \\
\chi(\mathcal{E}(-2))=4-\frac{1}{2}\left(3 c_{2}-c_{3}\right), \\
\chi(\mathcal{E}(-1))=10-\frac{1}{2}\left(5 c_{2}-c_{3}\right) .
\end{gathered}
$$

By taking into account the vanishing (3.2), we also have

$$
\begin{gathered}
h^{0}(\mathcal{E})=19-\frac{1}{2}\left(7 c_{2}-c_{3}\right)+r, \\
h^{0}(\mathcal{E}(1))=31-\frac{1}{2}\left(9 c_{2}-c_{3}\right)+4 r .
\end{gathered}
$$

Recall here that $H(\mathcal{E})^{r+2}=c_{3}-2 c_{1} c_{2}+c_{1}^{3}$ (see [6, $\S 3.1$ and $\left.\S 3.2\right]$ ). Since $c_{1}=3$ and $H(\mathcal{E})^{r+2} \geq 0$, this implies that

$$
c_{3} \geq 6 c_{2}-27 \text {. }
$$

\section{LEMMAS}

In this section, we collect some lemmas applied in the proofs of Theorems 1 or 3 .

Lemma 4.1. Let $V$ be an $(n+1)$-dimensional vector space, $\left(x_{0}, x_{1}, \ldots, x_{n}\right)$ a basis of $V$, and $\left(x_{0}^{*}, \ldots, x_{n}^{*}\right)$ the basis of the dual vector space $V^{*}$ corresponding to $\left(x_{0}, x_{1}, \ldots, x_{n}\right)$. Suppose that a non-zero element $s$ of $H^{0}\left(T_{\mathbb{P}(V)}(-1)\right)$ corresponds to $\sum_{i=0}^{n} a_{i} x_{i}^{*}$ for some $a_{i} \in K$ via the isomorphism from $H^{0}\left(T_{\mathbb{P}(V)}(-1)\right)$ to $V^{*}$. Then the zero locus $(s)_{0}$ of $s$ is a (reduced) point $p$ whose homogeneous coordinates $\left(x_{0}(p): \cdots: x_{n}(p)\right)$ are $\left(a_{0}: a_{1}: \cdots: a_{n}\right)$.

Proof. See [16, Remark 2.3].

Lemma 4.2. Let $i$ be any integer such that $1 \leq i \leq n$. For each non-zero morphism $\varphi: \Omega_{\mathbb{P}(V)}^{i}(i) \rightarrow \Omega_{\mathbb{P}(V)}^{i-1}(i-1)$, there exists a unique element $s \in H^{0}\left(T_{\mathbb{P}(V)}(-1)\right)$ such that $\varphi$ is the morphism appearing in the Koszul complex induced from s. In particular, every non-zero morphism $\varphi: \Omega_{\mathbb{P}^{3}}^{2}(2) \rightarrow \Omega_{\mathbb{P}^{3}}(1)$ determines a unique non-zero element $s \in H^{0}\left(T_{\mathbb{P}^{3}}(-1)\right)$ whose corresponding Koszul complex is an exact sequence

$$
0 \rightarrow \mathcal{O}(-1) \rightarrow \Omega_{\mathbb{P}^{3}}^{2}(2) \stackrel{\varphi}{\rightarrow} \Omega_{\mathbb{P}^{3}}(1) \rightarrow \mathcal{I}_{p} \rightarrow 0,
$$

where $\{p\}=(s)_{0}$.

Proof. We have an isomorphism $H^{0}\left(T_{\mathbb{P}(V)}(-1)\right) \cong \operatorname{Hom}\left(\Omega_{\mathbb{P}(V)}^{i}(i), \Omega_{\mathbb{P}(V)}^{i-1}(i-1)\right)$ by $[3$, Lemma 2], and this isomorphism indeed sends a global section $s$ of $T_{\mathbb{P}(V)}(-1)$ to the morphism $\varphi: \Omega_{\mathbb{P}(V)}^{i}(i) \rightarrow \Omega_{\mathbb{P}(V)}^{i-1}(i-1)$ appearing in the Koszul complex associated to $s$. 
Note that a non-zero section $s$ is regular by Lemma 4.1, so that the corresponding Koszul complex is exact.

Lemma 4.3. Let $W$ be a 0-dimensional closed subscheme of $\mathbb{P}^{2}$ and $w$ a point in $\mathbb{P}^{2}$. Suppose that we have the following non-split exact sequence of coherent sheaves

$$
0 \rightarrow \mathcal{I}_{W}(d) \rightarrow \mathcal{D} \rightarrow k(w) \rightarrow 0,
$$

where $d$ is an integer and $k(w)$ denotes the residue field of the point $w$. Then $w$ is an associated point of $W$ and $\mathcal{D}$ is isomorphic to $\mathcal{I}_{Z}(d)$ where $Z$ is a closed subscheme of $W$ with length $Z=$ length $W-1$.

Proof. First note that $w$ is not an associated point of $\mathcal{D}$, since the exact sequence above does not split. Hence $\mathcal{D}$ is a torsion-free sheaf of rank one, and its double dual is isomorphic to $\mathcal{O}(d)$. Therefore $\mathcal{D}$ is isomorphic to $\mathcal{I}_{Z}(d)$, where $Z$ is a closed subscheme of $W$ with length $Z=$ length $W-1$, and $w$ is an associated point of $W$.

Lemma 4.4. Let $Z$ be a 0 -dimensional closed subscheme of length 5 in $\mathbb{P}^{2}$, and suppose that length $(Z \cap L) \leq 2$ for any line $L$ in $\mathbb{P}^{2}$. Then there exists a smooth conic $C$ passing through $Z$.

Proof. See, e.g., the second paragraph of page 75 of [12].

\section{KeY LemmA}

The following lemma together with the exact sequence (3.16) plays a crucial role in the proofs of Theorems 1 and 3 .

Lemma 5.1. Let

$$
0 \rightarrow E_{2}^{-2,1} \rightarrow \mathcal{O}(-1)^{\oplus a} \stackrel{\mu}{\rightarrow} \Omega_{\mathbb{P}^{2}}(1)^{\oplus b} \rightarrow E_{2}^{-1,1} \rightarrow 0
$$

be the exact sequence 3.14) on $\mathbb{P}^{2}$, where $a=h^{1}(\mathcal{E}(-2))$ and $b=h^{1}(\mathcal{E}(-1))$.

(1) If $b \geq 1$, consider a surjection $\pi: \Omega_{\mathbb{P}^{2}}(1)^{\oplus b} \rightarrow \Omega_{\mathbb{P}^{2}}(1)$, and let

$$
\varphi: \mathcal{O}(-1)^{\oplus a} \rightarrow \Omega_{\mathbb{P}^{2}}(1)
$$

be the composite of $\mu$ and $\pi$. Then $H^{0}(\varphi(1)): H^{0}\left(\mathcal{O}^{\oplus a}\right) \rightarrow H^{0}\left(\Omega_{\mathbb{P}^{2}}(2)\right)$ is surjective; consequently $a \geq 3$ and $\varphi$ is surjective. Moreover we have an exact sequence

$$
0 \rightarrow E_{2}^{-2,1} \rightarrow \mathcal{O}(-2) \oplus \mathcal{O}(-1)^{\oplus a-3} \stackrel{\mu_{1}}{\rightarrow} \Omega_{\mathbb{P}^{2}}(1)^{\oplus b-1} \rightarrow E_{2}^{-1,1} \rightarrow 0 .
$$

(2) If $b \geq 2$, consider a surjection $q: \Omega_{\mathbb{P}^{2}}(1)^{\oplus b-1} \rightarrow \Omega_{\mathbb{P}^{2}}(1)$, and let

$$
\varphi_{1}: \mathcal{O}(-2) \oplus \mathcal{O}(-1)^{\oplus a-3} \rightarrow \Omega_{\mathbb{P}^{2}}(1)
$$

be the composite of $\mu_{1}$ and $q$. Then the image of $H^{0}\left(\varphi_{1}(1)\right)$ has dimension two or three; consequently $a \geq 5$.

(a) If $\operatorname{dim} \operatorname{Im} H^{0}\left(\varphi_{1}(1)\right)=2$, then we have the following exact sequence

$$
\begin{aligned}
0 \rightarrow E_{2}^{-2,1} \rightarrow \mathcal{O}(-3) \oplus \mathcal{O}(-1)^{\oplus a-5} & \stackrel{\nu_{2}}{\rightarrow} \Omega_{\mathbb{P}^{2}}(1)^{\oplus b-2} \\
& \rightarrow E_{2}^{-1,1} \rightarrow k(w) \rightarrow 0
\end{aligned}
$$

for some point $w$ in $\mathbb{P}^{2}$, where $k(w)$ denotes the residue field of $w$.

(b) If $\operatorname{dim} \operatorname{Im} H^{0}\left(\varphi_{1}(1)\right)=3$, then $a \geq 6$ and we have the following exact sequence

$$
0 \rightarrow E_{2}^{-2,1} \rightarrow \mathcal{O}(-2)^{\oplus 2} \oplus \mathcal{O}(-1)^{\oplus a-6} \stackrel{\mu_{2}}{\longrightarrow} \Omega_{\mathbb{P}^{2}}(1)^{\oplus b-2} \rightarrow E_{2}^{-1,1} \rightarrow 0 .
$$


Proof. (1) First note that there exists a surjection $E_{2}^{-1,1} \rightarrow$ Coker $\varphi$.

If $H^{0}(\varphi(1))=0$, then $\varphi=0$ and Coker $\varphi \cong \Omega_{\mathbb{P}^{2}}(1)$. This however contradicts Lemma 3.1. Therefore $H^{0}(\varphi(1)) \neq 0$.

Suppose that the image of $H^{0}(\varphi(1))$ has dimension one, and let $s$ be a non-zero element in the image in $H^{0}\left(\Omega_{\mathbb{P}^{2}}(2)\right) \cong H^{0}\left(T_{\mathbb{P}^{2}}(-1)\right)$. Then the zero locus of $s$ is a (reduced) point $p$ by Lemma 4.1 and Coker $\varphi$ is isomorphic to the ideal sheaf $\mathcal{I}_{p}$ of $p$, but this can not occur by Lemma 3.1. Therefore the image of $H^{0}(\varphi(1))$ has dimension $\geq 2$.

Suppose that the image of $H^{0}(\varphi(1))$ has dimension two, and let $(s, t)$ be a basis of the image. As above, let $p$ be the zero locus of $s$. Then $t$ induces an injection $\mathcal{O} \rightarrow \mathcal{I}_{p}(1)$, and this gives a line $L$ passing through $p$. The quotient of the $\mathcal{O}(-1)$-twist of this injection is $\mathcal{O}_{L}(-p)$ and is isomorphic to Coker $\varphi$. However this is impossible by Lemma 3.1. Therefore the image of $H^{0}(\varphi(1))$ has dimension $\geq 3$, i.e., $H^{0}(\varphi(1))$ is surjective. Hence $\varphi$ is surjective and $\operatorname{Ker} \varphi \cong \mathcal{O}(-2) \oplus \mathcal{O}(-1)^{\oplus a-3}$. Now Ker $\pi \cong \Omega_{\mathbb{P}^{2}}(1)^{\oplus b-1}$, and the morphism $\mu_{1}: \operatorname{Ker} \varphi \rightarrow \operatorname{Ker} \pi$ induced by $\mu$ extends to the exact sequence in the statement.

(2) Note first that there exists a surjection $E_{2}^{-1,1} \rightarrow$ Coker $\varphi_{1}$. Denote by $\iota$ the inclusion $\mathcal{O}(-1)^{\oplus a-3} \rightarrow \mathcal{O}(-2) \oplus \mathcal{O}(-1)^{\oplus a-3}$, and consider the composite $\varphi_{1} \circ \iota: \mathcal{O}(-1)^{\oplus a-3} \rightarrow$ $\Omega_{\mathbb{P}^{2}}(1)$. We see that $\varphi_{1}$ induces a morphism $\bar{\varphi}_{1}: \operatorname{Coker}(\iota) \rightarrow \operatorname{Coker}\left(\varphi_{1} \circ \iota\right)$ and that $\operatorname{Coker}(\iota) \cong \mathcal{O}(-2)$. Moreover we have the following long exact sequence

$$
0 \rightarrow \operatorname{Ker}\left(\varphi_{1} \circ \iota\right) \rightarrow \operatorname{Ker} \varphi_{1} \rightarrow \mathcal{O}(-2) \stackrel{\bar{\varphi}_{1}}{\longrightarrow} \operatorname{Coker}\left(\varphi_{1} \circ \iota\right) \rightarrow \operatorname{Coker} \varphi_{1} \rightarrow 0
$$

by the snake lemma. In particular, Coker $\varphi_{1} \cong \operatorname{Coker} \bar{\varphi}_{1}$.

If $H^{0}\left(\varphi_{1}(1)\right)=0$, then $\varphi_{1} \circ \iota=0$ and thus $\operatorname{Coker}\left(\varphi_{1} \circ \iota\right) \cong \Omega_{\mathbb{P}^{2}}(1)$. If $\bar{\varphi}_{1}=0$, then $\operatorname{Coker} \varphi_{1} \cong \operatorname{Coker}\left(\varphi_{1} \circ \iota\right)$. Hence $E_{2}^{-1,1}$ has $\Omega_{\mathbb{P}^{2}}(1)$ as a quotient, which is absurd by Lemma 3.1. Therefore $\bar{\varphi}_{1}: \mathcal{O}(-2) \rightarrow \Omega_{\mathbb{P}^{2}}(1)$ is a non-zero morphism. Suppose that $\bar{\varphi}_{1}$ is decomposed as $\bar{\varphi}_{1}=s l$, where $l$ is an inclusion $\mathcal{O}(-2) \rightarrow \mathcal{O}(-1)$ and $s$ is a nonzero morphism $\mathcal{O}(-1) \rightarrow \Omega_{\mathbb{P}^{2}}(1)$. The cokernel of the inclusion $l: \mathcal{O}(-2) \rightarrow \mathcal{O}(-1)$ is isomorphic to $\mathcal{O}_{L}(-1)$, where $L$ is the line defined by $l$, and the cokernel of the morphism $s: \mathcal{O}(-1) \rightarrow \Omega_{\mathbb{P}^{2}}(1)$ is isomorphic to $\mathcal{I}_{p}$, where $p$ is a point; thus Coker $\bar{\varphi}_{1}$ fits in an exact sequence

$$
0 \rightarrow \mathcal{O}_{L}(-1) \rightarrow \text { Coker } \bar{\varphi}_{1} \rightarrow \mathcal{I}_{p} \rightarrow 0
$$

Therefore Coker $\varphi_{1}$ (and hence $E_{2}^{-1,1}$ ) has $\mathcal{I}_{p}$ as a quotient, which can not happen by Lemma 3.1. Hence $\bar{\varphi}_{1}$ does not factor as $\bar{\varphi}_{1}=s l$. Let $\bar{s}$ be the non-zero section of $H^{0}\left(\Omega_{\mathbb{P}^{2}}(3)\right)$ determined by $\bar{\varphi}_{1}$. Then $\bar{s}$ is a regular section of $\Omega_{\mathbb{P}^{2}}(3) \cong T_{\mathbb{P}^{2}}$, i.e., the zero locus $Z$ of $\bar{s}$ has dimension $\leq 0$. Since $c_{2}\left(T_{\mathbb{P}^{2}}\right)=3, Z$ is thereby a 0 -dimensional closed subscheme of length three. Note that Coker $\bar{\varphi}_{1} \cong \mathcal{I}_{Z}(1)$; this however contradicts Lemma 3.1. Therefore $H^{0}\left(\varphi_{1}(1)\right) \neq 0$.

Suppose that the image of $H^{0}\left(\varphi_{1}(1)\right)$ has dimension one. Then $\operatorname{Coker}\left(\varphi_{1} \circ \iota\right)$ is isomorphic to the ideal sheaf $\mathcal{I}_{p}$ of some point $p$, as is shown in the proof of $(1)$. If $\bar{\varphi}_{1}=0$, then Coker $\bar{\varphi}_{1} \cong \mathcal{I}_{p}$, which is absurd by Lemma 3.1. Hence $\bar{\varphi}_{1}: \mathcal{O}(-2) \rightarrow \mathcal{I}_{p}$ is non-zero. Then Coker $\bar{\varphi}_{1}$ is isomorphic to $\mathcal{O}_{C}(-p)$ for some conic $C$ on $\mathbb{P}^{2}$, which again contradicts Lemma 3.1. Therefore the image of $H^{0}\left(\varphi_{1}(1)\right)$ has dimension at least two.

(2) (a) Suppose that $\operatorname{dim} \operatorname{Im} H^{0}\left(\varphi_{1}(1)\right)=2$. Then $\operatorname{Coker}\left(\varphi_{1} \circ \iota\right) \cong \mathcal{O}_{L}(-p)$ for some line $L$ in $\mathbb{P}^{2}$ and some point $p$ in $L$, as is shown in the proof of (1). If $\bar{\varphi}_{1}=0$, then Coker $\bar{\varphi}_{1} \cong \mathcal{O}_{L}(-p)$, which can not happen by Lemma 3.1. Hence $\bar{\varphi}_{1}: \mathcal{O}(-2) \rightarrow \mathcal{O}_{L}(-p)$ is non-zero. Then Coker $\bar{\varphi}_{1} \cong k(w)$ for some point $w$ on $\mathbb{P}^{2}$, and $\operatorname{Ker} \bar{\varphi}_{1} \cong \mathcal{O}(-3)$. The 
long exact sequence (5.1) induces an exact sequence

$$
0 \rightarrow \operatorname{Ker}\left(\varphi_{1} \circ \iota\right) \rightarrow \operatorname{Ker} \varphi_{1} \rightarrow \operatorname{Ker} \bar{\varphi}_{1} \rightarrow 0 .
$$

Note here that $\operatorname{Ker} \varphi_{1} \circ \iota \cong \mathcal{O}(-1)^{\oplus a-5}$; the above sequence then implies that $\operatorname{Ker} \varphi_{1} \cong$ $\mathcal{O}(-3) \oplus \mathcal{O}(-1)^{\oplus a-5}$. Now we get the exact sequence in the statement by the snake lemma.

(2) (b) Suppose that $\operatorname{dim} \operatorname{Im} H^{0}\left(\varphi_{1}(1)\right) \geq 3$. Then $H^{0}\left(\varphi_{1}(1)\right)$ is surjective, and thus $\varphi_{1} \circ \iota$ is surjective. Hence $\operatorname{Ker} \varphi_{1} \circ \iota \cong \mathcal{O}(-2) \oplus \mathcal{O}(-1)^{\oplus a-6}$. Then the long exact sequence (5.1) gives an exact sequence

$$
0 \rightarrow \mathcal{O}(-2) \oplus \mathcal{O}(-1)^{\oplus a-6} \rightarrow \operatorname{Ker} \varphi_{1} \rightarrow \mathcal{O}(-2) \rightarrow 0 .
$$

Therefore $\operatorname{Ker} \varphi_{1} \cong \mathcal{O}(-2)^{\oplus 2} \oplus \mathcal{O}(-1)^{\oplus a-6}$. Now Ker $q \cong \Omega_{\mathbb{P}^{2}}(1)^{\oplus b-2}$, and $\mu_{1}$ induces a morphism $\operatorname{Ker} \varphi_{1} \rightarrow \operatorname{Ker} q$, which extends to the exact sequence in the statement.

\section{Global generation of $\mathcal{E}$ in Case $c_{2} \leq 7$}

Theorem 3. Let $\mathcal{E}$ be a nef vector bundle of rank $r$ on a projective space $\mathbb{P}^{n}$ with first Chern class $c_{1}=3$ and second Chern class $c_{2} \leq 7$. Then $\mathcal{E}$ is globally generated.

Proof. Consider first the case $n=2$. Note that $\mathcal{E}$ is 0-regular in the sense of CastelnuovoMumford if $h^{1}(\mathcal{E}(-1))=0$ since we have the vanishing (3.4). Since 0-regularity implies global generation, we may concentrate our attention to the case $h^{1}(\mathcal{E}(-1)) \neq 0$. We shall work in the setting in $\$ 3.1$ to apply the Bondal spectral sequence (2.1). Then it follows from Lemma 5.1 (1) and formula (3.10) that $c_{2} \geq 6$. Moreover the assumption $c_{2} \leq 7$ implies that

$$
h^{1}(\mathcal{E}(-1))=1
$$

by Lemma 5.1 (2). Lemma 5.1 (1) then shows that $E_{2}^{-1,1}=0$. Therefore $E_{3}^{0,0} \cong \mathcal{E}$ by the exact sequence (3.16). Note that $E_{3}^{0,0}$ is a quotient of $E_{2}^{0,0}$ since $E_{2}^{p, q}=0$ if $p>0$. Since $E_{2}^{0,0}$ is globally generated by (3.17), this shows that $\mathcal{E}$ is globally generated if $n=2$ and $c_{2} \leq 7$.

Consider next the case $n \geq 3$. Recall here that $\mathcal{E}$ is globally generated if $h^{1}(\mathcal{E}(-1))=0$ and $\left.\mathcal{E}\right|_{H}$ is globally generated for any hyperplane $H$ in $\mathbb{P}^{n}$ by [15, Lemma 3].

Suppose now that $n=3$. Since $\left.\mathcal{E}\right|_{H}$ is globally generated for any plane $H$ as we have seen above, we may concentrate our attention to the case $h^{1}(\mathcal{E}(-1)) \neq 0$. Then it follows from (3.3) that $H(\mathcal{E})$ is not big, and hence $c_{3}=6 c_{2}-27$ by (3.23). Thus $c_{3}$ is odd. Hence $c_{2}$ is odd by (3.20). Since $c_{3}$ is non-negative by (3.7), we see that $c_{2} \geq 5$ by equality $c_{3}=6 c_{2}-27$; thus $\left(c_{2}, c_{3}\right)=(5,3)$ or $(7,15)$. Suppose that $\left(c_{2}, c_{3}\right)=(7,15)$. Then $h^{1}\left(\left.\mathcal{E}\right|_{H}(-1)\right)=1$ by (6.1) . Hence $h^{0}\left(\left.\mathcal{E}\right|_{H}(-1)\right)=0$ by (3.11). Since $h^{0}(\mathcal{E}(-2))=0$ by (3.1), this implies that $h^{0}(\mathcal{E}(-1))=0$. Note here that $H^{q}(\mathcal{E})=0$ for all $q>0$ by (3.2) and that $H^{q}\left(\left.\mathcal{E}\right|_{H}\right)=0$ for all $q>0$ by (3.8). Hence $H^{q}(\mathcal{E}(-1))=0$ unless $q=1$. Thus

$$
-h^{1}(\mathcal{E}(-1))=\chi(\mathcal{E}(-1))=\left(c_{3}-15\right) / 2=0
$$

by (3.20). This contradicts the assumption $h^{1}(\mathcal{E}(-1)) \neq 0$; thus the case where $\left(c_{2}, c_{3}\right)=$ $(7,15)$ is ruled out. In $\$ 6.1$, we shall show that the case $\left(c_{2}, c_{3}\right)=(5,3)$ does not happen. Therefore $h^{1}(\mathcal{E}(-1))=0$ and $\mathcal{E}$ is globally generated.

If $n \geq 4$, then $h^{1}(\mathcal{E}(-1))=0$ by $(3.2)$ and $\left.\mathcal{E}\right|_{H}$ is globally generated for any hyperplane $H$ in $\mathbb{P}^{n}$ as we have seen above. Therefore $\mathcal{E}$ is globally generated. 
6.1. The case where $\left(n, c_{2}, c_{3}\right)=(3,5,3)$. We first claim that

$$
\begin{aligned}
H^{q}\left(\left.\mathcal{E}\right|_{L^{2}}(-k)\right) & =0 \quad \text { for all } q>0 \text { and all } k \leq 2 \text { unless }(q, k)=(1,2) \\
h^{1}\left(\left.\mathcal{E}\right|_{L^{2}}(-2)\right) & =2,
\end{aligned}
$$

where $L^{2}$ denotes a plane in $\mathbb{P}^{3}$. Indeed we have $h^{1}\left(\left.\mathcal{E}\right|_{L^{2}}(-2)\right)=2$ by $(\underline{3.10})$, and $H^{q}\left(\left.\mathcal{E}\right|_{L^{2}}(-k)\right)$ vanishes for any $k \leq 0$ and any $q>0$ by (3.8), for $(k, q)=(1,2)$ and $(2,2)$ by $(3.4)$, and for $(k, q)=(1,1)$ by Lemma $5.1(1)$ and $(3.10)$. Since $H^{q}(\mathcal{E}(-k))=0$ for all $q>0$ if $k \leq 0$ by (3.2), the claims above imply that

$$
\begin{aligned}
H^{q}(\mathcal{E}(-k)) & =0 \text { for all } q \geq 2 \text { and all } k \leq 3 \text { unless }(q, k)=(2,3) \\
h^{2}(\mathcal{E}(-3)) & \leq 2
\end{aligned}
$$

Set

$$
a=h^{2}(\mathcal{E}(-3))
$$

Then

$$
a=h^{1}(\mathcal{E}(-3))
$$

by (3.1), (3.18), and (6.4). It follows from (3.1), (3.19), and (6.4) that

$$
h^{1}(\mathcal{E}(-2))=2 .
$$

We also have

$$
\chi(\mathcal{E}(-1))=-1
$$

by (3.20). By (3.21), we have

$$
h^{0}(\mathcal{E})=r+3
$$

Note that there exists an exact sequence

$$
0 \rightarrow H^{0}(\mathcal{E}(-1)) \rightarrow H^{0}\left(\left.\mathcal{E}\right|_{L^{2}}(-1)\right) \rightarrow H^{1}(\mathcal{E}(-2)) \rightarrow H^{1}(\mathcal{E}(-1)) \rightarrow 0
$$

and that $h^{0}\left(\left.\mathcal{E}\right|_{L^{2}}(-1)\right)=1$ by $(\underline{3.11})$ and (6.2) . Therefore we have two cases, corresponding to $h^{0}(\mathcal{E}(-1))=0$ or 1 . If $h^{0}(\mathcal{E}(-1))=1$, then $h^{1}(\mathcal{E}(-1))=2$, and we shall consider the following exact sequence

$$
0 \rightarrow \mathcal{O}(1) \rightarrow \mathcal{E} \rightarrow \mathcal{F} \rightarrow 0
$$

of coherent sheaves on $\mathbb{P}^{3}$. Note here that $\operatorname{Ext}^{q}(G, \mathcal{E}) \cong \operatorname{Ext}^{q}(G, \mathcal{F})$ if $q>0$, that $\operatorname{Hom}(\mathcal{O}(i), \mathcal{F})=0$ if $i \geq 2$, that $\operatorname{Hom}(\mathcal{O}(1), \mathcal{F})=0$ by the assumption $h^{0}(\mathcal{E}(-1))=1$, and that $\operatorname{hom}(\mathcal{O}, \mathcal{F})=r-1$ by $(\underline{6.9})$.

6.1.1. Set-up using the Bondal spectral sequence. Suppose that $\left(h^{0}(\mathcal{E}(-1)), h^{1}(\mathcal{E}(-1))\right)=$ $(0,1)$ (resp. $(1,2))$. We apply to $\mathcal{E}$ (resp. $\mathcal{F}$ ) the Bondal spectral sequence (2.1). It follows from (6.4) that $E_{2}^{p, q}=0$ for all $p$ if $q \geq 3$. We have $\operatorname{Ext}^{2}(G, \mathcal{E}) \cong S_{3}^{\oplus a}$ by (6.4) and (6.6). Hence $E_{2}^{p, 2}=0$ unless $p=-3$, and $E_{2}^{-3,2}=\mathcal{O}(-1)^{\oplus a}$ by (2.5). It follows from (6.7), (6.8), $(3.2)$, and the assumption $h^{1}(\mathcal{E}(-1))=1$ (resp. 2) that the right $A$-module $\operatorname{Ext}^{1}(G, \mathcal{E})$ 
(resp. $\left.\operatorname{Ext}^{1}(G, \mathcal{F})\right)$ has the filtration which induces the following exact sequences of right $A$-modules

$$
\begin{aligned}
0 \rightarrow F & \rightarrow \operatorname{Ext}^{1}(G, \mathcal{E}) \rightarrow S_{3}^{\oplus a} \rightarrow 0 ; \\
0 & \rightarrow S_{1} \rightarrow F \rightarrow S_{2}^{\oplus 2} \rightarrow 0 . \\
(\text { resp. } 0 & \rightarrow F \rightarrow \operatorname{Ext}^{1}(G, \mathcal{F}) \rightarrow S_{3}^{\oplus a} \rightarrow 0 ; \\
0 & \left.\rightarrow S_{1}^{\oplus 2} \rightarrow F \rightarrow S_{2}^{\oplus 2} \rightarrow 0 .\right)
\end{aligned}
$$

Correspondingly we obtain the following distinguished triangles

$$
\begin{gathered}
F \otimes^{\mathrm{L}}{ }_{A} G \rightarrow \operatorname{Ext}^{1}(G, \mathcal{E}) \otimes^{\mathrm{L}}{ }_{A} G \rightarrow \mathcal{O}(-1)^{\oplus a}[3] \rightarrow ; \\
\Omega_{\mathbb{P}^{3}}(1)[1] \rightarrow F \otimes^{\mathrm{L}}{ }_{A} G \rightarrow \Omega_{\mathbb{P}^{3}}^{2}(2)^{\oplus 2}[2] \rightarrow \\
\text { (resp. } F \otimes^{\mathrm{L}}{ }_{A} G \rightarrow \operatorname{Ext}^{1}(G, \mathcal{F}) \otimes^{\mathrm{L}}{ }_{A} G \rightarrow \mathcal{O}(-1)^{\oplus a}[3] \rightarrow ; \\
\left.\Omega_{\mathbb{P}^{3}}(1)^{\oplus 2}[1] \rightarrow F \otimes^{\mathrm{L}}{ }_{A} G \rightarrow \Omega_{\mathbb{P}^{3}}^{2}(2)^{\oplus 2}[2] \rightarrow\right)
\end{gathered}
$$

by (2.4). We see that $\mathcal{H}^{-1}\left(F \otimes^{\mathrm{L}}{ }_{A} G\right) \cong E_{2}^{-1,1}$ by (6.11) (resp. (6.13)). Moreover we have $\mathcal{H}^{p}\left(F \otimes{ }_{A}^{\mathrm{L}} G\right)=0$ unless $p=-2$ or -1 by (6.12) (resp.(6.14)). Set $\mathcal{M}=\mathcal{H}^{-2}\left(F \otimes{ }^{\mathrm{L}}{ }_{A} G\right)$. Then it follows from (6.11) and (6.12) (resp. (6.13) and (6.14)) that $\mathcal{M}$ fits in the following exact sequences

$$
\begin{gathered}
0 \rightarrow E_{2}^{-3,1} \rightarrow \mathcal{O}(-1)^{\oplus a} \rightarrow \mathcal{M} \rightarrow E_{2}^{-2,1} \rightarrow 0 ; \\
0 \rightarrow \mathcal{M} \rightarrow \Omega_{\mathbb{P}^{3}}^{2}(2)^{\oplus 2} \stackrel{\varphi}{\rightarrow} \Omega_{\mathbb{P}^{3}}(1) \rightarrow E_{2}^{-1,1} \rightarrow 0 . \\
\left(\text { resp. } 0 \rightarrow \mathcal{M} \rightarrow \Omega_{\mathbb{P}^{3}}^{2}(2)^{\oplus 2} \stackrel{\varphi}{\rightarrow} \Omega_{\mathbb{P}^{3}}(1)^{\oplus 2} \rightarrow E_{2}^{-1,1} \rightarrow 0 .\right)
\end{gathered}
$$

It follows from (6.9) and the assumption $h^{0}(\mathcal{E}(-1))=0$ (resp. 1) that the right $A$-module $\operatorname{Hom}(G, \mathcal{E})(\operatorname{resp} . \operatorname{Hom}(G, \mathcal{F}))$ is isomorphic to $S_{0}^{\oplus r+3}\left(\right.$ resp. $\left.S_{0}^{\oplus r-1}\right)$. Hence $E_{2}^{p, 0}=0$ unless $p=0$, and $E_{2}^{0,0}$ is isomorphic to $\mathcal{O}_{\mathbb{P} 3}^{\oplus r+3}$ (resp. $\mathcal{O}_{\mathbb{P} 3}^{\oplus r-1}$ ) by $(2.4)$. Therefore $E_{2}^{p, q}=0$ unless $(p+q, q)=(0,0),(0,1),(-1,1),(-1,2)$, or $(-2,1)$. Then it follows from (2.1) that $E_{2}^{-3,1}=0$, and consequently the sequence (6.15) becomes the following exact sequence

$$
0 \rightarrow \mathcal{O}(-1)^{\oplus a} \rightarrow \mathcal{M} \rightarrow E_{2}^{-2,1} \rightarrow 0 .
$$

Besides (6.16) (resp. (6.17)) and (6.18), the spectral sequence (2.1) induces the following exact sequences

$$
\begin{gathered}
0 \rightarrow E_{2}^{-2,1} \rightarrow \mathcal{O}_{\mathbb{P}^{3}}^{\oplus r+3} \rightarrow E_{3}^{0,0} \rightarrow 0 ; \\
\left(\text { resp. } 0 \rightarrow E_{2}^{-2,1} \rightarrow \mathcal{O}_{\mathbb{P}^{3}}^{\oplus r-1} \rightarrow E_{3}^{0,0} \rightarrow 0 ;\right) \\
0 \rightarrow E_{3}^{-3,2} \rightarrow \mathcal{O}(-1)^{\oplus a} \rightarrow E_{2}^{-1,1} \rightarrow E_{3}^{-1,1} \rightarrow 0 ; \\
0 \rightarrow E_{3}^{-3,2} \rightarrow E_{3}^{0,0} \rightarrow E_{4}^{0,0} \rightarrow 0 ; \\
0 \rightarrow E_{4}^{0,0} \rightarrow \mathcal{E} \rightarrow E_{3}^{-1,1} \rightarrow 0 . \\
\left(\text { resp. } 0 \rightarrow E_{4}^{0,0} \rightarrow \mathcal{F} \rightarrow E_{3}^{-1,1} \rightarrow 0 .\right)
\end{gathered}
$$

For $i=1$ (resp. $i=1,2$ ) and $j=1,2$, denote by $\varphi_{i j}$ the composite of the $j$-th inclusion $\Omega_{\mathbb{P}^{3}}^{2}(2) \hookrightarrow \Omega_{\mathbb{P}^{3}}^{2}(2)^{\oplus 2}$ and the morphism $\varphi$ appearing in the exact sequence (6.16) (resp. (6.17) ) and the $i$-th projection.

We claim here that $\varphi_{11}$ and $\varphi_{12}$ (resp. $\varphi_{i 1}$ and $\varphi_{i 2}$ for each $i$, and $\varphi_{1 j}$ and $\varphi_{2 j}$ for each j) are linearly independent in $\operatorname{Hom}\left(\Omega_{\mathbb{P}^{3}}^{2}(2), \Omega_{\mathbb{P}^{3}}(1)\right)$. Indeed, if they are not, then we may 
assume that $\varphi_{12}=0$ by some row or column elementary transformations; thus $\varphi$ (resp. the composite of $\varphi$ and the first projection $\left.\Omega_{\mathbb{P}^{3}}(1)^{\oplus 2} \rightarrow \Omega_{\mathbb{P}^{3}}(1)\right)$ factors through $\varphi_{11}$ via the first projection $\Omega_{\mathbb{P}^{3}}^{2}(2)^{\oplus 2} \rightarrow \Omega_{\mathbb{P}^{3}}^{2}(2)$. Note here that the cokernel of $\varphi_{11}$ is isomorphic to the ideal sheaf $\mathcal{I}_{p}$ for some point $p \in \mathbb{P}^{3}$ by Lemma 4.2 if $\varphi_{11} \neq 0$ and to $\Omega_{\mathbb{P}^{3}}(1)$ if $\varphi_{11}=0$. Hence there exists a surjection $E_{2}^{-1,1} \rightarrow \mathcal{I}_{p}$ by (6.16) (resp. (6.17)) (even if $\left.\varphi_{11}=0\right)$. Consider the composite of the morphism $\mathcal{O}(-1)^{\oplus a} \rightarrow E_{2}^{-1,1}$ in (6.21) and the surjection $E_{2}^{-1,1} \rightarrow \mathcal{I}_{p}$. Since the cokernel of the composite is a quotient of $E_{3}^{-1,1}$ and $a \leq 2$ by (6.5) and (6.6) , we see that $E_{3}^{-1,1}$ has $\mathcal{O}_{L}(-1)$ as a quotient for some line $L$ passing through $p$ in $\mathbb{P}^{3}$. On the other hand, $E_{3}^{-1,1}$ can not have a negative degree sheaf as a quotient, since it is a quotient of the nef vector bundle $\mathcal{E}$ by (6.23) (resp. (6.24) and $(6.10))$. This is a contradiction. Therefore the claim holds.

In case $\left(h^{0}(\mathcal{E}(-1)), h^{1}(\mathcal{E}(-1))\right)=(1,2)$, denote by $V$ the vector space generated by $\varphi_{i j}(1 \leq i, j \leq 2)$ in the following. If $\operatorname{dim} V \leq 3$, then the claim above implies that there exist $\lambda_{i j} \in K(1 \leq i, j \leq 2)$ such that $\lambda_{21} \varphi_{11}+\lambda_{22} \varphi_{21}=\lambda_{11} \varphi_{12}+\lambda_{12} \varphi_{22} \neq 0$. Therefore, by changing the free basis consisting two elements of $\Omega_{\mathbb{P}^{3}}(1)^{\oplus 2}$, we may assume that $\varphi_{21}=\varphi_{12}$ in case $\operatorname{dim} V \leq 3$. Moreover if $\operatorname{dim} V=2$, we can make $\varphi_{12}$ to be zero after some row and column elementary transformations, which contradicts the claim above. Therefore $\operatorname{dim} V=3$ or 4 , and we may furthermore assume that $\varphi_{11}, \varphi_{12}$, and $\varphi_{22}$ are linearly independent.

Set $\varphi_{0}=\varphi_{11}$ and $\varphi_{1}=\varphi_{12}$. (resp. Set $\varphi_{0}=\varphi_{11}, \varphi_{1}=\varphi_{12}, \varphi_{2}=\varphi_{21}$, and $\varphi_{3}=\varphi_{22}$. Note that $\varphi_{1}=\varphi_{2}$ in case $\operatorname{dim} V=3$.)

Let $e_{i}^{*}$ be the image of $x_{i}^{*}$ via the isomorphism $H^{0}(\mathcal{O}(1))^{*} \rightarrow H^{0}\left(T_{\mathbb{P}^{3}}(-1)\right)$. Let $s$ be an element of $H^{0}\left(T_{\mathbb{P}^{3}}(-1)\right)$, and suppose that $s=\sum_{i=0}^{3} a_{i} e_{i}^{*}$ for some $a_{i} \in K$. Note that $e_{0}^{*}=-\sum_{i=1}^{3}\left(x_{i} / x_{0}\right) e_{i}^{*}$ and $\left(e_{1}^{*}, e_{2}^{*}, e_{3}^{*}\right)$ is a local basis of $T_{\mathbb{P}^{3}}(-1)$ over $D_{+}\left(x_{0}\right)$, where $D_{+}\left(x_{0}\right)$ is the open set defined by $x_{0} \neq 0$. Thus $s=\sum_{i=1}^{3}\left\{a_{i}-a_{0}\left(x_{i} / x_{0}\right)\right\} e_{i}^{*}$ over $D_{+}\left(x_{0}\right)$. Let $\left(e_{1}, e_{2}, e_{3}\right)$ be the dual basis of $\left.\Omega_{\mathbb{P}^{3}}(1)\right|_{D_{+}\left(x_{0}\right)}$. Then the Koszul morphism $\varphi_{s}$ from $\Omega_{\mathbb{P}^{3}}^{2}(2)$ to $\Omega_{\mathbb{P}^{3}}(1)$ corresponding to the section $s$ has the representation matrix

$$
\left[\begin{array}{ccc}
0 & a_{3}-a_{0}\left(x_{3} / x_{0}\right) & -\left(a_{2}-a_{0}\left(x_{2} / x_{0}\right)\right) \\
-\left(a_{3}-a_{0}\left(x_{3} / x_{0}\right)\right) & 0 & a_{1}-a_{0}\left(x_{1} / x_{0}\right) \\
a_{2}-a_{0}\left(x_{2} / x_{0}\right) & -\left(a_{1}-a_{0}\left(x_{1} / x_{0}\right)\right) & 0
\end{array}\right]
$$

with respect to the bases $\left(e_{2} \wedge e_{3}, e_{3} \wedge e_{1}, e_{1} \wedge e_{2}\right)$ and $\left(e_{1}, e_{2}, e_{3}\right)$ over $D_{+}\left(x_{0}\right)$. Denote this matrix by $M\left(a_{0}, a_{1}, a_{2}, a_{3}\right)$.

Now Lemma 4.2 enables us to assume, by taking homogeneous coordinates $\left(x_{0}: x_{1}: x_{2}: x_{3}\right)$ of $\mathbb{P}^{3}$ suitably, that $\varphi_{i}$ corresponds to the global section $e_{i}^{*}$ of $T_{\mathbb{P}^{3}}(-1)$ for $i=0,1$. Similarly, in case $\left(h^{0}(\mathcal{E}(-1)), h^{1}(\mathcal{E}(-1))\right)=(1,2)$, we may assume that $\varphi_{i}$ corresponds to the global section $e_{i}^{*}$ of $T_{\mathbb{P}^{3}}(-1)$ for $i=0,1,2,3$ if $\operatorname{dim} V=4$ and that $\varphi_{i}$ to $e_{i}^{*}$ except $i=2$ and $\varphi_{2}$ to $e_{1}^{*}$ if $\operatorname{dim} V=3$.

We claim here that $\operatorname{coker}\left(\left[\varphi_{0}, \varphi_{1}\right]\right): \Omega_{\mathbb{P}^{3}}(1) \rightarrow \operatorname{Coker}\left(\left[\varphi_{0}, \varphi_{1}\right]\right)$ is the composite of the two restrictions $\left.\Omega_{\mathbb{P}^{3}}(1) \rightarrow \Omega_{\mathbb{P}^{3}}(1)\right|_{L}$ and $\left.\Omega_{\mathbb{P}^{3}}(1)\right|_{L} \rightarrow \Omega_{L}(1)$ where $L$ is the line defined by $x_{2}=x_{3}=0$. Note first that the morphism $\left[\varphi_{0}, \varphi_{1}\right]: \Omega_{\mathbb{P}^{3}}^{2}(2)^{\oplus 2} \rightarrow \Omega_{\mathbb{P}^{3}}(1)$ has the representation matrix

$$
[M(1,0,0,0) \quad M(0,1,0,0)]=\left[\begin{array}{cccccc}
0 & -x_{3} / x_{0} & x_{2} / x_{0} & 0 & 0 & 0 \\
x_{3} / x_{0} & 0 & -x_{1} / x_{0} & 0 & 0 & 1 \\
-x_{2} / x_{0} & x_{1} / x_{0} & 0 & 0 & -1 & 0
\end{array}\right]
$$


with respect to the bases $\left(e_{2} \wedge e_{3}, e_{3} \wedge e_{1}, e_{1} \wedge e_{2}, e_{2}^{\prime} \wedge e_{3}^{\prime}, e_{3}^{\prime} \wedge e_{1}^{\prime}, e_{1}^{\prime} \wedge e_{2}^{\prime}\right)$ and $\left(e_{1}, e_{2}, e_{3}\right)$ over $D_{+}\left(x_{0}\right)$, where $\left(e_{1}^{\prime}, e_{2}^{\prime}, e_{3}^{\prime}\right)$ is the dual basis of the second component $\left.\Omega_{\mathbb{P}^{3}}(1)\right|_{D_{+}\left(x_{0}\right)}$ of the direct sum $\Omega_{\mathbb{P}^{3}}^{2}(2)^{\oplus 2}$. Since $e_{i}=x_{0} d\left(x_{i} / x_{0}\right)$ for $i=1,2,3$, the image $\left.\operatorname{Im}\left[\varphi_{0}, \varphi_{1}\right]\right|_{D_{+}\left(x_{0}\right)}$ of $\left.\left[\varphi_{0}, \varphi_{1}\right]\right|_{D_{+}\left(x_{0}\right)}$ is generated by $x_{0} d\left(x_{2} / x_{0}\right), x_{0} d\left(x_{3} / x_{0}\right), x_{2} d\left(x_{1} / x_{0}\right)$, and $x_{3} d\left(x_{1} / x_{0}\right)$. Note that $x_{2} d\left(x_{i} / x_{0}\right)=\left(x_{2} / x_{0}\right) x_{0} d\left(x_{i} / x_{0}\right)$ and $x_{3} d\left(x_{i} / x_{0}\right)=\left(x_{3} / x_{0}\right) x_{0} d\left(x_{i} / x_{0}\right) \quad(1 \leq$ $i \leq 3)$ and that the quotient of $\left.\Omega_{\mathbb{P}^{3}}(1)\right|_{D_{+}\left(x_{0}\right)}$ by the submodule generated by $x_{2} d\left(x_{i} / x_{0}\right)$ and $x_{3} d\left(x_{i} / x_{0}\right)(1 \leq i \leq 3)$ is $\left.\left(\Omega_{\mathbb{P}^{3}}(1) \otimes \mathcal{O}_{L}\right)\right|_{D_{+}\left(x_{0}\right)}$, where $L$ is the line defined by $x_{2}=x_{3}=0$. Hence Coker $\left.\left(\left[\varphi_{0}, \varphi_{1}\right]\right)\right|_{D_{+}\left(x_{0}\right)}$ is nothing but the quotient $\left.\Omega_{L}(1)\right|_{D_{+}\left(x_{0}\right)}$ of $\left.\left(\Omega_{\mathbb{P}^{3}}(1) \otimes \mathcal{O}_{L}\right)\right|_{D_{+}\left(x_{0}\right)}$ by the submodule generated by $x_{0} d\left(x_{2} / x_{0}\right) \otimes 1$ and $x_{0} d\left(x_{3} / x_{0}\right) \otimes$ 1. Similarly $\left.\operatorname{Coker}\left(\left[\varphi_{0}, \varphi_{1}\right]\right)\right|_{D_{+}\left(x_{1}\right)}$ is naturally isomorphic to $\left.\Omega_{L}(1)\right|_{D_{+}\left(x_{1}\right)}$. Moreover $\left.\operatorname{Coker}\left(\left[\varphi_{0}, \varphi_{1}\right]\right)\right|_{D_{+}\left(x_{i}\right)}=0$ for $i=2$ and 3 . Therefore the claim holds.

6.1.2. The case where $\left(h^{0}(\mathcal{E}(-1)), h^{1}(\mathcal{E}(-1))\right)=(0,1)$. Since $\varphi=\left[\varphi_{0}, \varphi_{1}\right]$, we see by the claim above that $\operatorname{coker}(\varphi): \Omega_{\mathbb{P}^{3}}(1) \rightarrow E_{2}^{-1,1}$ is nothing but the composite $\Omega_{\mathbb{P}^{3}}(1) \rightarrow$ $\left.\Omega_{\mathbb{P}^{3}}(1)\right|_{L} \rightarrow \Omega_{L}(1)$ of the restrictions, where $L$ is a line. Therefore $E_{2}^{-1,1} \cong \mathcal{O}_{L}(-1)$. Then the sequence (6.21) implies that $E_{3}^{-1,1}$ is either $\mathcal{O}_{L}(-1)$ or zero, but (6.23) and the nefness of $\mathcal{E}$ imply that the former can not happen. Therefore $E_{3}^{-1,1}=0$ and $E_{4}^{0,0} \cong \mathcal{E}$; thus we obtain a surjection $\mathcal{O}^{\oplus r+3} \rightarrow \mathcal{E}$ by (6.19) and (6.22). In fact, this can not happen, as can be seen below.

Denote by $\mathcal{H}$ the kernel of the surjection $\mathcal{O}^{\oplus r+3} \rightarrow \mathcal{E} ; \mathcal{H}$ is a vector bundle of rank three. Note here that the dual $\mathcal{H}^{\vee}$ of $\mathcal{H}$ is globally generated. Moreover $c_{1}\left(\mathcal{H}^{\vee}\right)=3$ and

$$
c_{2}\left(\mathcal{H}^{\vee}\right)=c_{2}(\mathcal{H})=-c_{1}(\mathcal{H}) c_{1}-c_{2}=4 .
$$

Furthermore

$$
c_{3}\left(\mathcal{H}^{\vee}\right)=-c_{3}(\mathcal{H})=c_{2}(\mathcal{H}) c_{1}+c_{1}(\mathcal{H}) c_{2}+c_{3}=0 .
$$

Since $\mathcal{H}^{\vee}$ is a globally generated vector bundle of rank three with $c_{3}\left(\mathcal{H}^{\vee}\right)=0$, we have an exact sequence of vector bundles

$$
0 \rightarrow \mathcal{O} \rightarrow \mathcal{H}^{\vee} \rightarrow \mathcal{H}^{\prime} \rightarrow 0
$$

Then it follows from [5, Proposition 2.4] that $\mathcal{H}^{\prime} \cong \mathcal{O}(1) \oplus \mathcal{O}(2)$. This however contradicts that $c_{2}\left(\mathcal{H}^{\vee}\right)=4$. The case $\left(h^{0}(\mathcal{E}(-1)), h^{1}(\mathcal{E}(-1))\right)=(0,1)$ thus can not happen.

6.1.3. The case where $\left(h^{0}(\mathcal{E}(-1)), h^{1}(\mathcal{E}(-1))\right)=(1,2)$. As we have shown in $\$ 6.1 .1$, the composite $\left[\varphi_{0}, \varphi_{1}\right]$ of $\varphi$ and the first projection $\Omega_{\mathbb{P}^{3}}(1)^{\oplus 2} \rightarrow \Omega_{\mathbb{P}^{3}}(1)$ has $\Omega_{L}(1)$ as its cokernel, where $L$ is the line defined by $x_{2}=x_{3}=0$. Therefore $E_{2}^{-1,1}$ has $\mathcal{O}_{L}(-1)$ as a quotient by (6.17).

It follows from (6.25) that $\varphi$ has, with respect to the bases $\left(e_{2} \wedge e_{3}, e_{3} \wedge e_{1}, e_{1} \wedge e_{2}, e_{2}^{\prime} \wedge\right.$ $\left.e_{3}^{\prime}, e_{3}^{\prime} \wedge e_{1}^{\prime}, e_{1}^{\prime} \wedge e_{2}^{\prime}\right)$ and $\left(e_{1}, e_{2}, e_{3}, e_{1}^{\prime}, e_{2}^{\prime}, e_{3}^{\prime}\right)$ over $D_{+}\left(x_{0}\right)$, the representation matrix

$$
\left[\begin{array}{ll}
M(1,0,0,0) & M(0,1,0,0) \\
M(0,0,1,0) & M(0,0,0,1)
\end{array}\right]
$$

if $\operatorname{dim} V=4$, and the representation matrix

$$
\left[\begin{array}{ll}
M(1,0,0,0) & M(0,1,0,0) \\
M(0,1,0,0) & M(0,0,0,1)
\end{array}\right]
$$

if $\operatorname{dim} V=3$. Therefore $\operatorname{Coker}(\varphi)$ is supported on the quadric surface defined by $x_{0} x_{3}-$ $x_{1} x_{2}=0$ if $\operatorname{dim} V=4$ and by $x_{2}^{2}=0$ if $\operatorname{dim} V=3$. This implies that $\varphi$ is injective. Hence $\mathcal{M}=0$ by (6.17); it follows from (6.18) that $a=0$ and $E_{2}^{-2,1}=0$; thus $E_{2}^{-1,1} \cong E_{3}^{-1,1}$ by 
(6.21). Since $E_{2}^{-1,1}$ has $\mathcal{O}_{L}(-1)$ as a quotient, so does $E_{3}^{-1,1}$. However $E_{3}^{-1,1}$ can not have $\mathcal{O}_{L}(-1)$ as a quotient by (6.10) and (6.24) since $\mathcal{E}$ is nef. Therefore we conclude that the case $\left(h^{0}(\mathcal{E}(-1)), h^{1}(\mathcal{E}(-1))\right)=(1,2)$ does not happen.

\section{Proof of Theorem 1}

As we stated at the end of $₫ \mathbb{1}$, we always assume that $c_{2}=9$ in this section.

7.1. Proof for the case $n=2$ and $h^{1}(\mathcal{E})=0$. It follows from (3.10), (3.11), and (3.12) that

$$
\begin{gathered}
h^{1}(\mathcal{E}(-2))=6, \\
h^{0}(\mathcal{E}(-1))-h^{1}(\mathcal{E}(-1))=-3, \\
h^{0}(\mathcal{E})=r .
\end{gathered}
$$

As in $\oint 3.1$, set $e_{0,1}=h^{0}(\mathcal{E}(-1))$. Then

$$
e_{0,1} \leq 1
$$

The proof of this fact goes almost identical to that in case $c_{2}=8$, i.e., that in [12, $\left.\S 3.1\right]$ : what we have to do is just to change several numerals related to $c_{2}=8$ to those related to $c_{2}=9$; so we omit the proof of this fact.

We apply to $\mathcal{E}$ the Bondal spectral sequence (2.1). As we have seen in $\$ 3.1$ and Lemma 5.1 (2), $E_{2}^{p, q}$ vanishes unless $(p, q)=(-2,1),(-1,1)$ or $(0,0)$, and $E_{2}^{-2,1}$ and $E_{2}^{-1,1}$ fit in one of the following exact sequences:

$$
\begin{aligned}
0 \rightarrow E_{2}^{-2,1} & \rightarrow \mathcal{O}(-3) \oplus \mathcal{O}(-1) \stackrel{\nu_{2}}{\rightarrow} \Omega_{\mathbb{P}^{2}}(1)^{\oplus e_{0,1}+1} \rightarrow E_{2}^{-1,1} \rightarrow k(w) \rightarrow 0 \\
0 & \rightarrow E_{2}^{-2,1} \rightarrow \mathcal{O}(-2)^{\oplus 2} \stackrel{\mu_{2}}{\rightarrow} \Omega_{\mathbb{P}^{2}}(1)^{\oplus e_{0,1}+1} \rightarrow E_{2}^{-1,1} \rightarrow 0
\end{aligned}
$$

where $k(w)$ denotes the residue field of some point $w$ in $\mathbb{P}^{2}$. Recall that each of the exact sequences is a consequence of the assumption $H^{1}(\mathcal{E})=0$. Note that $E_{2}^{0,0}$ fits in the following exact sequence by (3.17)

$$
0 \rightarrow \mathcal{O}^{\oplus 3 e_{0,1}} \rightarrow \mathcal{O}(1)^{\oplus e_{0,1}} \oplus \mathcal{O}^{\oplus r} \rightarrow E_{2}^{0,0} \rightarrow 0 .
$$

Lemma 7.1. If $E_{2}^{-2,1}=0$, then $E_{2}^{0,0} \cong E_{3}^{0,0} \cong \mathcal{O}(1)^{\oplus e_{0,1}} \oplus \mathcal{O}^{\oplus r-3 e_{0,1}}$, and $\mathcal{E}$ fits in the following exact sequence

$$
0 \rightarrow \mathcal{O}(1)^{\oplus e_{0,1}} \oplus \mathcal{O}^{\oplus r-3 e_{0,1}} \rightarrow \mathcal{E} \rightarrow E_{2}^{-1,1} \rightarrow 0 .
$$

Moreover $H^{i}\left(E_{2}^{-1,1}\right)=0$ for all $i$; thus $E_{2}^{-1,1}$ is the cokernel of the evaluation map $H^{0}(\mathcal{E}) \otimes$ $\mathcal{O} \rightarrow \mathcal{E}$

Proof. Since $E_{2}^{-2,1}=0, E_{2}^{0,0} \cong E_{3}^{0,0}$ by (3.15), and hence $E_{2}^{0,0}$ is torsion-free by (3.16). Therefore (7.7) shows that $\mathcal{O}(1)$ is a subsheaf of $E_{2}^{0,0}$ if $e_{0,1}=1$, and consequently we see that $E_{2}^{0,0}$ is isomorphic to $\mathcal{O}(1)^{\oplus e_{0,1}} \oplus \mathcal{O}^{\oplus r-3 e_{0,1}}$. Hence it follows from $(3.16)$ that $\mathcal{E}$ fits in the desired exact sequence (7.8). Since we have (17.3),$H^{0}\left(\mathcal{O}(1)^{\oplus e_{0,1}} \oplus \mathcal{O}^{\oplus r-3 e_{0,1}}\right)$ is isomorphic to $H^{0}(\mathcal{E})$, and thus $H^{0}\left(E_{2}^{-1,1}\right)$ vanishes. Furthermore $H^{i}\left(E_{2}^{-1,1}\right)=0$ for all $i$ since $h^{1}(\mathcal{E})=0$ by assumption and $h^{2}(\mathcal{E})=0$ by (3.4).

Let $\alpha_{i j}: \mathcal{O}(-2) \rightarrow \Omega_{\mathbb{P}^{2}}(1)$ be the composite of the $j$-th inclusion $\mathcal{O}(-2) \rightarrow \mathcal{O}(-2)^{\oplus 2}$, $\mu_{2}$, and the $i$-th projection $\Omega_{\mathbb{P}^{2}}(1)^{\oplus e_{0,1}+1} \rightarrow \Omega_{\mathbb{P}^{2}}(1)$. The $\alpha_{i j}$ 's form a matrix $\left[\alpha_{i j}\right]$, which is another expression of $\mu_{2}$. 
Lemma 7.2. For each $i, \alpha_{i 1}$ and $\alpha_{i 2}$ are linearly independent. Similarly, for each $j$, elements in the $j$-th column are linearly independent. Furthermore the cokernel of the composite $\left[\alpha_{11}, \alpha_{12}\right]$ of $\mu_{2}$ and the first projection $\Omega_{\mathbb{P}^{2}}(1)^{\oplus e_{0,1}+1} \rightarrow \Omega_{\mathbb{P}^{2}}(1)$ is supported on a cubic curve $E$, and $\left[\alpha_{11}, \alpha_{12}\right]$ and $\mu_{2}$ are injective. In particular $E_{2}^{-2,1}=0$ in (7.6).

Proof. First note that $E_{2}^{-1,1}$ in (17.6) has as a quotient the cokernel of the composite, say $\psi$, of $\mu_{2}$ and any surjection $\Omega_{\mathbb{P}^{2}}(1)^{e_{0,1}+1} \rightarrow \Omega_{\mathbb{P}^{2}}(1)$. Since $E_{2}^{-1,1}$ can not have $\Omega_{\mathbb{P}^{2}}(1)$ as a quotient by Lemma 3.1, we observe that the composite $\psi$ can not be zero.

Suppose that elements in one of the rows or the columns are linearly dependent. After some row or column elementary transformations, by replacing $\alpha_{i j}$, we may then assume that $\alpha_{11} \neq 0$ and that $\alpha_{12}=0$ by the observation above. The morphism $\left[\alpha_{11}, 0\right]$ factors through the first projection $\mathcal{O}(-2)^{\oplus 2} \rightarrow \mathcal{O}(-2)$, and $\operatorname{Coker}\left(\left[\alpha_{11}, 0\right]\right)=\operatorname{Coker}\left(\alpha_{11}\right)$. If $\alpha_{11}$ factors through $\mathcal{O}(-1)$, the cokernel of the induced morphism $\mathcal{O}(-1) \rightarrow \Omega_{\mathbb{P}^{2}}(1)$ is isomorphic to $\mathcal{I}_{p}$ for some point $p \in \mathbb{P}^{2}$ and $\operatorname{Coker}\left(\alpha_{11}\right)$ fits in the following exact sequence

$$
0 \rightarrow \mathcal{O}_{L}(-1) \rightarrow \operatorname{Coker}\left(\alpha_{11}\right) \rightarrow \mathcal{I}_{p} \rightarrow 0,
$$

where $\mathcal{O}_{L}(-1)$ is the cokernel of the induced injection $\mathcal{O}(-2) \rightarrow \mathcal{O}(-1)$. In particular $\mathcal{O}_{L}(-1)$ is the torsion subsheaf of $\operatorname{Coker}\left(\alpha_{11}\right)$, and $\mathcal{I}_{p}$ is the quotient of Coker $\left(\alpha_{11}\right)$ by its torsion subsheaf. This contradicts Lemma 3.1. Therefore $\alpha_{11}$ does not factor through $\mathcal{O}(-1)$; this implies that $\operatorname{Coker}\left(\alpha_{11}\right)$ is isomorphic to $\mathcal{I}_{Z}(1)$, where $Z$ is a 0 -dimensional closed subscheme of length three on $\mathbb{P}^{2}$. This however can not happen by Lemma 3.1 . Therefore elements in the same row or column are linearly independent.

Since $\alpha_{11}$ and $\alpha_{12}$ are linearly independent, the morphism $\left[\alpha_{11}, \alpha_{12}\right]$ induces a non-zero morphism $\bar{\alpha}_{12}: \mathcal{O}(-2) \rightarrow \operatorname{Coker}\left(\alpha_{11}\right)$, and we see that $\operatorname{Coker}\left(\left[\alpha_{11}, \alpha_{12}\right]\right) \cong \operatorname{Coker}\left(\bar{\alpha}_{12}\right)$. Denote by $\operatorname{Coker}\left(\alpha_{11}\right) /$ tors the quotient of $\operatorname{Coker}\left(\alpha_{11}\right)$ by its torsion subsheaf. As we have seen above, $\operatorname{Coker}\left(\alpha_{11}\right) /$ tors is either $\mathcal{I}_{p}$ or $\mathcal{I}_{Z}(1)$. If $\operatorname{Coker}\left(\alpha_{11}\right) /$ tors $=\mathcal{I}_{p}$, then Coker $\left(\bar{\alpha}_{12}\right)$ admits a negative degree line bundle on a conic as a quotient, which is impossible by Lemma 3.1. Hence Coker $\left(\alpha_{11}\right)=\mathcal{I}_{Z}(1)$; thus Coker $\left(\bar{\alpha}_{12}\right)$ is supported on a cubic curve $E$ passing through $Z$. Therefore $\bar{\alpha}_{12}$ is injective. Since $\alpha_{11}$ is also injective, so is $\left[\alpha_{11}, \alpha_{12}\right]$. Hence $\mu_{2}$ is injective, and thus $E_{2}^{-2,1}=0$.

Lemma 7.3. If $E_{2}^{-1,1}$ fits in the exact sequence (7.6), then it fits in the following exact sequence

$$
0 \rightarrow \mathcal{O}(-2)^{\oplus e_{0,1}+3} \rightarrow \mathcal{O}(-1)^{\oplus 3 e_{0,1}+3} \rightarrow E_{2}^{-1,1} \rightarrow 0 .
$$

Proof. By Lemma $7.2, E_{2}^{-2,1}=0$, and thus $E_{2}^{-1,1}$ fits in the following exact sequence

$$
0 \rightarrow \mathcal{O}(-2)^{\oplus 2} \stackrel{\mu_{2}}{\longrightarrow} \Omega_{\mathbb{P}^{2}}(1)^{\oplus e_{0,1}+1} \rightarrow E_{2}^{-1,1} \rightarrow 0 .
$$

Since $\Omega_{\mathbb{P}^{2}}(1)$ is isomorphic to $T_{\mathbb{P}^{2}}(-2)$, the Euler sequence and the sequence above induce the desired exact sequence (7.9).

Denote by $\mathcal{K}$ the kernel of the surjection $E_{2}^{-1,1} \rightarrow k(w)$ in (7.5) .

Lemma 7.4. The sheaf $\mathcal{K}$ can not admit the following sheaves as a quotient:

(1) $\Omega_{\mathbb{P}^{2}}(1)$;

(2) $\mathcal{I}_{W}(1)$, where $W$ is a 0 -dimensional closed subscheme of length $W \geq 3$;

(3) $\mathcal{I}_{W}(2)$, where $W$ is a 0 -dimensional closed subscheme of length $W \geq 6$. 
Proof. If $\mathcal{K}$ admits $\Omega_{\mathbb{P}^{2}}(1)$ as a quotient, then $\left.E_{2}^{-1,1}\right|_{L}$ admits $\mathcal{O}_{L}(-1)$ as a quotient for a line $L$ not passing through $w$. This contradicts Lemma 3.1. Suppose that $\mathcal{K}$ admits $\mathcal{I}_{W}(d)$ as a quotient, where length $W \geq 3$ if $d=1$ and length $W \geq 6$ if $d=2$. Then we obtain the following exact sequence

$$
0 \rightarrow \mathcal{I}_{W}(d) \rightarrow \mathcal{D} \rightarrow k(w) \rightarrow 0
$$

for some quotient sheaf $\mathcal{D}$ of $E_{2}^{-1,1}$. Note that this sequence does not split since $E_{2}^{-1,1}$ does not admit $\mathcal{I}_{W}(d)$ as a quotient by Lemma 3.1. Lemma 4.3 then implies that $\mathcal{D} \cong \mathcal{I}_{Z}(d)$, where $Z$ is a 0 -dimensional closed subscheme of length $Z=\operatorname{length} W-1$. However $E_{2}^{-1,1}$ can not admit $\mathcal{I}_{Z}(d)$ as a quotient by Lemma 3.1.

Let $\beta_{i}: \mathcal{O}(-1) \rightarrow \Omega_{\mathbb{P}^{2}}(1)$ be the composite of the inclusion $\mathcal{O}(-1) \rightarrow \mathcal{O}(-3) \oplus \mathcal{O}(-1)$, $\nu_{2}$, and the $i$-th projection $\Omega_{\mathbb{P}^{2}}(1)^{\oplus e_{0,1}+1} \rightarrow \Omega_{\mathbb{P}^{2}}(1)$, and let $\gamma_{i}: \mathcal{O}(-3) \rightarrow \Omega_{\mathbb{P}^{2}}(1)$ be the composite of the inclusion $\mathcal{O}(-3) \rightarrow \mathcal{O}(-3) \oplus \mathcal{O}(-1), \nu_{2}$, and the $i$-th projection $\Omega_{\mathbb{P}^{2}}(1)^{\oplus e_{0,1}+1} \rightarrow \Omega_{\mathbb{P}^{2}}(1)$. We shall denote by $\operatorname{Coker}\left(\gamma_{i}\right) /$ tors the quotient of $\operatorname{Coker}\left(\gamma_{i}\right)$ by its torsion subsheaf.

Lemma 7.5. If $\gamma_{i} \neq 0$, then $\operatorname{Coker}\left(\gamma_{i}\right) /$ tors is isomorphic to $\mathcal{I}_{W}(d)$ for some 0 -dimensional closed subscheme $W$ of $\mathbb{P}^{2}$ and some integer $d$, and the possible values of the pair (length $W, d$ ) are $(7,2),(3,1)$, or $(1,0)$.

Proof. Suppose that $\gamma_{i}$ factors through $\mathcal{O}(-1)$. The cokernel of the induced morphism $\mathcal{O}(-1) \rightarrow \Omega_{\mathbb{P}^{2}}(1)$ is isomorphic to $\mathcal{I}_{p}$ for some point $p \in \mathbb{P}^{2}$, and $\operatorname{Coker}\left(\gamma_{i}\right)$ fits in the following exact sequence

$$
0 \rightarrow \mathcal{O}_{C}(-1) \rightarrow \operatorname{Coker}\left(\gamma_{i}\right) \rightarrow \mathcal{I}_{p} \rightarrow 0,
$$

where $C$ is a conic and $\mathcal{O}_{C}(-1)$ is the cokernel of the induced morphism $\mathcal{O}(-3) \rightarrow \mathcal{O}(-1)$. Hence (length $W, d)=(1,0)$ in this case.

Suppose that $\gamma_{i}$ does not factor through $\mathcal{O}(-1)$ and that it factors through $\mathcal{O}(-2)$. Then the induced morphism $\mathcal{O}(-2) \rightarrow \Omega_{\mathbb{P}^{2}}(1)$ does not factor through $\mathcal{O}(-1)$ and the cokernel of the morphism $\mathcal{O}(-2) \rightarrow \Omega_{\mathbb{P}^{2}}(1)$ is isomorphic to $\mathcal{I}_{Z}(1)$ for some closed subscheme $Z \subset \mathbb{P}^{2}$ of length three. Moreover $\operatorname{Coker}\left(\gamma_{i}\right)$ fits in the following exact sequence

$$
0 \rightarrow \mathcal{O}_{L}(-2) \rightarrow \operatorname{Coker}\left(\gamma_{i}\right) \rightarrow \mathcal{I}_{Z}(1) \rightarrow 0,
$$

where $L$ is the line determined by the induced morphism $\mathcal{O}(-3) \rightarrow \mathcal{O}(-2)$ and $\mathcal{O}_{L}(-2)$ is the cokernel of that morphism. Hence (length $W, d)=(3,1)$ in this case.

Finally suppose that $\gamma_{i}$ does not factor through $\mathcal{O}(-1)$ nor $\mathcal{O}(-2)$. Then $\gamma_{i}$ induces a regular section $s \in H^{0}\left(\Omega_{\mathbb{P}^{2}}(4)\right)$, whose zero locus $(s)_{0}$ has length seven. Hence Coker $\left(\gamma_{i}\right)$ is isomorphic to $\mathcal{I}_{W}(2)$, where $W=(s)_{0}$. Therefore (length $\left.W, d\right)=(7,2)$ in this case.

Lemma 7.6. For any $i, \beta_{i}$ is non-zero.

Proof. Suppose, to the contrary, that $\beta_{1}=0$. Then the composite $\left[\gamma_{1}, \beta_{1}\right]$ of $\nu_{2}$ and the first projection $\Omega_{\mathbb{P}^{2}}(1)^{e_{0,1}+1} \rightarrow \Omega_{\mathbb{P}^{2}}(1)$ factors through $\mathcal{O}(-3)$ via the projection $\mathcal{O}(-3) \oplus$ $\mathcal{O}(-1) \rightarrow \mathcal{O}(-3)$, and $\operatorname{Coker}\left(\left[\gamma_{1}, 0\right]\right)$ is isomorphic to $\operatorname{Coker}\left(\gamma_{1}\right)$. Note that $\operatorname{Coker}\left(\gamma_{1}\right)$ is a quotient of $\mathcal{K}$. It follows from Lemma 7.4 that $\gamma_{1} \neq 0$. Then $\operatorname{Coker}\left(\gamma_{1}\right) /$ tors $\cong \mathcal{I}_{W}(d)$ for some 0 -dimensional closed subscheme $W$ of $\mathbb{P}^{2}$, and the possible values of the pair (length $W, d)$ are $(7,2),(3,1)$, or $(1,0)$ by Lemma 7.5 . However the values $(7,2)$ and $(3,1)$ are ruled out by Lemma 7.4. Hence (length $W, d)=(1,0)$ and thus the torsion subsheaf 
of $\operatorname{Coker}\left(\gamma_{1}\right)$ is isomorphic to $\mathcal{O}_{C}(-1)$, where $C$ is a conic on $\mathbb{P}^{2}$. Set $\{p\}=W$. There exists the following commutative diagram with exact rows

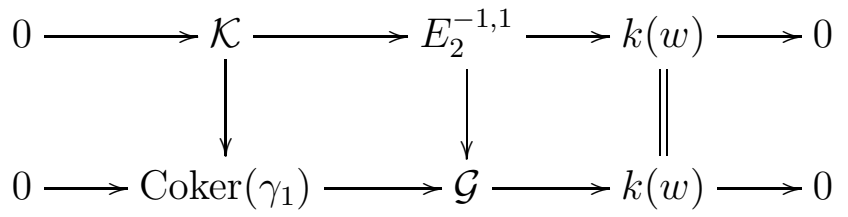

for some quotient coherent sheaf $\mathcal{G}$ of $E_{2}^{-1,1}$. Denote by $\mathcal{D}$ the cokernel of the composite of the two inclusions $\mathcal{O}_{C}(-1) \rightarrow \operatorname{Coker}\left(\gamma_{1}\right)$ and $\operatorname{Coker}\left(\gamma_{1}\right) \rightarrow \mathcal{G}$. Then we have the following commutative diagram with exact rows.

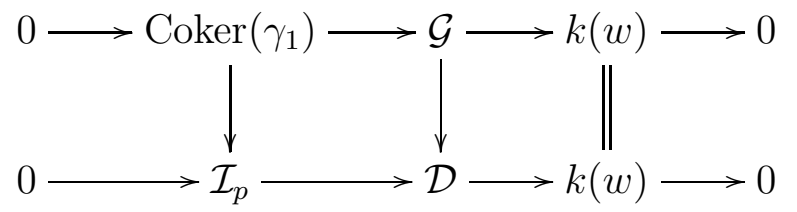

Note here that the bottom row of the diagram above does not split since $E_{2}^{-1,1}$ can not admit $\mathcal{I}_{p}$ as a quotient by Lemma 3.1. Therefore Lemma 4.3 implies that $w=p$ and that $\mathcal{D}$ is isomorphic to $\mathcal{O}_{\mathbb{P}^{2}}$. Hence $\mathcal{G}$ fits in the following exact sequence

$$
0 \rightarrow \mathcal{O}_{C}(-1) \rightarrow \mathcal{G} \rightarrow \mathcal{O}_{\mathbb{P}^{2}} \rightarrow 0 .
$$

Let $\mathcal{E}_{1}$ be the kernel of the composite of the two surjections $\mathcal{E} \rightarrow \mathcal{G}$ and $\mathcal{G} \rightarrow \mathcal{O}_{\mathbb{P}^{2}}$. Then $\mathcal{O}_{C}(-1)$ is a quotient of $\mathcal{E}_{1}$. Hence $\left.\mathcal{E}_{1}\right|_{C}$ admits $\mathcal{O}_{C}(-1)$ as a quotient, and it also fits in the following exact sequence

$$
\left.\left.0 \rightarrow \mathcal{E}_{1}\right|_{C} \rightarrow \mathcal{E}\right|_{C} \rightarrow \mathcal{O}_{C} \rightarrow 0
$$

Since $C$ is a conic, this implies that $\left.\mathcal{E}\right|_{C}$ admits a negative degree quotient, which contradicts Lemma 3.1 .

There exists the natural composition

$$
\operatorname{Hom}\left(\mathcal{O}(-1), \Omega_{\mathbb{P}^{2}}(1)\right) \times \operatorname{Hom}(\mathcal{O}(-3), \mathcal{O}(-1)) \rightarrow \operatorname{Hom}\left(\mathcal{O}(-3), \Omega_{\mathbb{P}^{2}}(1)\right),
$$

and we have two cases:

- $\gamma_{1}$ has $\beta_{1}$ as a factor;

- $\gamma_{1}$ does not have $\beta_{1}$ as a factor.

Lemma 7.7. If $\gamma_{1}$ does not have $\beta_{1}$ as a factor, then $E_{2}^{-2,1}=0$ and $E_{2}^{-1,1}$ fits in (7.8) and 7.9 .

Proof. If $\gamma_{1}$ does not have $\beta_{1}$ as a factor, then $\left[\gamma_{1}, \beta_{1}\right]$ induces a non-zero morphism $\bar{\gamma}_{1}: \mathcal{O}(-3) \rightarrow \operatorname{Coker}\left(\beta_{1}\right)$. Since $\beta_{1} \neq 0$ by Lemma [7.6, this implies that $\left[\gamma_{1}, \beta_{1}\right]$ is injective. Hence $\nu_{2}$ is injective and thus $E_{2}^{-2,1}=0$. Lemma 7.1 then shows that $E_{2}^{-1,1}$ lies in (7.8). Since $E_{2}^{-2,1}=0, \mathcal{K}$ fits in the following exact sequence

$$
0 \rightarrow \mathcal{O}(-3) \oplus \mathcal{O}(-1) \rightarrow \Omega_{\mathbb{P}^{2}}(1)^{\oplus e_{0,1}+1} \rightarrow \mathcal{K} \rightarrow 0 .
$$

This together with the isomorphism $\Omega_{\mathbb{P}^{2}}(1) \cong T_{\mathbb{P}^{2}}(-2)$ and the Euler sequence induces the following exact sequence

$$
0 \rightarrow \mathcal{O}(-3) \oplus \mathcal{O}(-2)^{\oplus e_{0,1}+1} \rightarrow \mathcal{O}(-1)^{\oplus 3 e_{0,1}+2} \rightarrow \mathcal{K} \rightarrow 0 .
$$


Since we have an exact sequence

$$
0 \rightarrow \mathcal{O}(-3) \rightarrow \mathcal{O}(-2)^{\oplus 2} \rightarrow \mathcal{O}(-1) \rightarrow k(w) \rightarrow 0,
$$

the resolution of $\mathcal{K}$ above implies that $E_{2}^{-1,1}$ fits in the following exact sequence

$$
0 \rightarrow \mathcal{O}(-3) \stackrel{d_{2}}{\rightarrow} \mathcal{O}(-3) \oplus \mathcal{O}(-2)^{\oplus e_{0,1}+3} \rightarrow \mathcal{O}(-1)^{\oplus 3 e_{0,1}+3} \rightarrow E_{2}^{-1,1} \rightarrow 0 .
$$

Lemma 7.1 and this sequence (7.12) then show that $H^{2}\left(d_{2}\right)$ is an isomorphism; we infer that the composite of $d_{2}$ and the projection $\mathcal{O}(-3) \oplus \mathcal{O}(-2)^{\oplus e_{0,1}+3} \rightarrow \mathcal{O}(-3)$ is an isomorphism. Therefore the exact sequence (7.12) is reduced to the exact sequence (7.9).

Now if $E_{2}^{-1,1}$ lies in (7.6) or if $E_{2}^{-1,1}$ lies in (7.5) and $\gamma_{1}$ does not have $\beta_{1}$ as a factor, then $E_{2}^{-1,1}$ lies also in (17.8) and (7.9) by Lemmas 17.1, 7.2, 7.3, and 7.7. Hence if $e_{0,1}=1$ then we obtain the following exact sequences:

$$
\begin{gathered}
0 \rightarrow \mathcal{O}(-2)^{\oplus 4} \rightarrow \mathcal{O}(-1)^{\oplus 6} \rightarrow E_{2}^{-1,1} \rightarrow 0 ; \\
0 \rightarrow \mathcal{O}(1) \oplus \mathcal{O}^{\oplus r-3} \rightarrow \mathcal{E} \rightarrow E_{2}^{-1,1} \rightarrow 0 .
\end{gathered}
$$

These two exact sequences imply that $\mathcal{E}$ fits in the exact sequence in case (18) of Theorem 1. If $e_{0,1}=0$ then we obtain the following exact sequences:

$$
\begin{gathered}
0 \rightarrow \mathcal{O}(-2)^{\oplus 3} \rightarrow \mathcal{O}(-1)^{\oplus 3} \rightarrow E_{2}^{-1,1} \rightarrow 0 ; \\
0 \rightarrow \mathcal{O}^{\oplus r} \rightarrow \mathcal{E} \rightarrow E_{2}^{-1,1} \rightarrow 0 .
\end{gathered}
$$

These two exact sequences show that $\mathcal{E}$ fits in the exact sequence in case (16) of Theorem 1 , Question 1. Does there exist a nef vector bundle fitting in the exact sequence in case (18) of Theorem 1 ?

Remark 7.1. If $E_{2}^{-1,1}$ is in (7.15), then $E_{2}^{-1,1}$ is supported on a cubic curve.

Lemma 7.8. If $\gamma_{1}$ has $\beta_{1}$ as a factor, then $e_{0,1}=0, E_{2}^{-1,1} \cong \mathcal{O}$, and we have the following non-split exact sequences:

$$
\begin{gathered}
0 \rightarrow \mathcal{O}(-3) \rightarrow \mathcal{O}^{\oplus r} \rightarrow E_{3}^{0,0} \rightarrow 0 ; \\
0 \rightarrow E_{3}^{0,0} \rightarrow \mathcal{E} \rightarrow \mathcal{O} \rightarrow 0 .
\end{gathered}
$$

In particular $E_{2}^{-1,1}$ is the cokernel of the evaluation map $H^{0}(\mathcal{E}) \otimes \mathcal{O} \rightarrow \mathcal{E}$.

Proof. Since $\gamma_{1}$ has $\beta_{1}$ as a factor, by changing the free basis of $\mathcal{O}(-3) \oplus \mathcal{O}(-1)$, we may assume that $\gamma_{1}=0$.

Suppose that $e_{0,1}=1$. Then we have the following commutative diagram with exact rows.

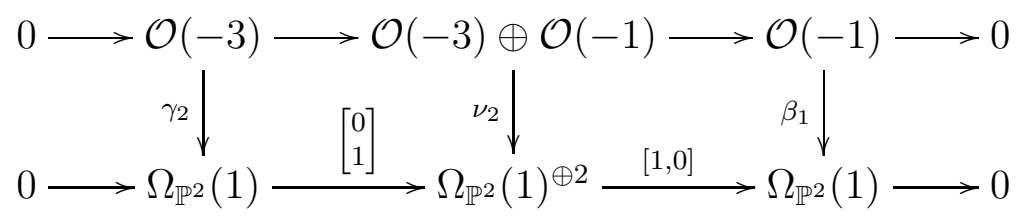

Since $\beta_{1} \neq 0$ by Lemma 7.6, $\beta_{1}$ is injective and $\operatorname{Coker}\left(\beta_{1}\right)=\mathcal{I}_{p}$ for some point $p \in \mathbb{P}^{2}$. Hence the diagram above induces an exact sequence

$$
0 \rightarrow \operatorname{Coker}\left(\gamma_{2}\right) \rightarrow \mathcal{K} \rightarrow \mathcal{I}_{p} \rightarrow 0
$$


Denote by $\mathcal{D}$ the quotient of $E_{2}^{-1,1}$ by $\operatorname{Coker}\left(\gamma_{2}\right)$. Then we obtain the following commutative diagram with exact rows.

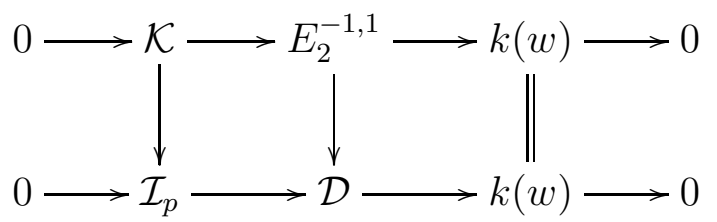

Since $E_{2}^{-1,1}$ can not admit $\mathcal{I}_{p}$ as a quotient by Lemma 3.1, the bottom row of the diagram above does not split. Hence $p=w$ and $\mathcal{D}$ is isomorphic to $\mathcal{O}_{\mathbb{P}^{2}}$ by Lemma 4.3. The definition of $\mathcal{D}$ induces the following exact sequence

$$
0 \rightarrow \operatorname{Coker}\left(\gamma_{2}\right) \rightarrow E_{2}^{-1,1} \rightarrow \mathcal{O} \rightarrow 0 .
$$

If $\gamma_{2}=0$, then $\operatorname{Coker}\left(\gamma_{2}\right) \cong \Omega_{\mathbb{P}^{2}}(1)$, and thus $E_{2}^{-1,1} \cong \Omega_{\mathbb{P}^{2}}(1) \oplus \mathcal{O}$, but this is absurd by Lemma 3.1. Therefore $\gamma_{2} \neq 0$, and it follows from Lemma 7.5 that Coker $\left(\gamma_{2}\right) /$ tors is isomorphic to $\mathcal{I}_{W}(d)$ for some 0-dimensional closed subscheme $W$ of $\mathbb{P}^{2}$ and that the possible values of the pair (length $W, d)$ are $(7,2),(3,1)$, or $(1,0)$. The sequence above induces an exact sequence

$$
0 \rightarrow \mathcal{I}_{W}(d) \rightarrow E_{2}^{-1,1} / \text { tors } \rightarrow \mathcal{O} \rightarrow 0
$$

Now Lemma 4.4 implies that there exists a smooth rational curve $C$ such that the $d$-th twist $\mathcal{I}_{W} \cdot \mathcal{O}_{C}(d)$ of the inverse image ideal sheaf $\mathcal{I}_{W} \cdot \mathcal{O}_{C}$ is a negative degree line bundle. This implies that $\left.E_{2}^{-1,1}\right|_{C}$ admits a negative degree line bundle as a direct summand, which contradicts Lemma 3.1, Therefore the case $e_{0,1}=1$ can not happen.

Suppose that $e_{0,1}=0$. Since $\beta_{1} \neq 0$ by Lemma 7.6 and $\gamma_{1}=0$ by assumption, the exact sequence (7.5) shows that $E_{2}^{-2,1} \cong \mathcal{O}(-3)$ and that $\mathcal{K} \cong \operatorname{Coker}\left(\beta_{1}\right) \cong \mathcal{I}_{p}$. Since the sequence

$$
0 \rightarrow \mathcal{I}_{p} \rightarrow E_{2}^{-1,1} \rightarrow k(w) \rightarrow 0
$$

does not split by Lemma 3.1, we see that $p=w$ and that

$$
E_{2}^{-1,1} \cong \mathcal{O}_{\mathbb{P}^{2}}
$$

by Lemma 4.3. Note here that $E_{2}^{0,0} \cong \mathcal{O}^{\oplus r}$ by (7.7). Hence we obtain the desired exact sequences (7.17) and (7.18) by (3.15) and (3.16). Finally the sequence (7.18) does not split since $H^{0}\left(E_{3}^{0,0}\right) \cong H^{0}(\mathcal{E})$ by (7.3) and (7.17) .

Suppose that $\gamma_{1}$ has $\beta_{1}$ as a factor. We shall give a resolution of $\mathcal{E}$ in terms of a full strong exceptional collection $(\mathcal{O}(-3), \mathcal{O}(-2), \mathcal{O}(-1))$ of line bundles on $\mathbb{P}^{2}$. By composing the Euler exact sequence and the dual of that, we obtain the following exact sequence

$$
0 \rightarrow \mathcal{O}(-3) \rightarrow \mathcal{O}(-2)^{\oplus 3} \rightarrow \mathcal{O}(-1)^{\oplus 3} \rightarrow \mathcal{O} \rightarrow 0 .
$$

Hence we obtain the following exact sequence

$$
0 \rightarrow \mathcal{O}(-3)^{\oplus r} \rightarrow \mathcal{O}(-2)^{\oplus 3 r} \rightarrow \mathcal{O}(-1)^{\oplus 3 r} \rightarrow \mathcal{O}^{\oplus r} \rightarrow 0 .
$$

The exact sequence (7.17) then induces the following exact sequence

$$
0 \rightarrow \mathcal{O}(-3)^{\oplus r} \rightarrow \mathcal{O}(-2)^{\oplus 3 r} \oplus \mathcal{O}(-3) \rightarrow \mathcal{O}(-1)^{\oplus 3 r} \rightarrow E_{3}^{0,0} \rightarrow 0 .
$$

The sequence (7.18) together with (7.20) and (7.19) induces the following resolution of $\mathcal{E}$ :

$$
0 \rightarrow \mathcal{O}(-3)^{\oplus r+1} \stackrel{d_{2}}{\rightarrow} \mathcal{O}(-2)^{\oplus 3 r+3} \oplus \mathcal{O}(-3) \rightarrow \mathcal{O}(-1)^{\oplus 3 r+3} \rightarrow \mathcal{E} \rightarrow 0 .
$$


Since $h^{1}(\mathcal{E})=0$ by assumption, the linear map $H^{2}\left(d_{2}\right)$ is surjective. Hence the composite of $d_{2}$ and the projection $\mathcal{O}(-2)^{\oplus 3 r+3} \oplus \mathcal{O}(-3) \rightarrow \mathcal{O}(-3)$ is non-zero and thus a surjection. Therefore the resolution above is reduced to that in case (17) of Theorem 11. This completes the proof of Theorem 1 for the case $n=2, c_{2}=9$, and $h^{0}(\mathcal{E})=0$.

Here we give the following two remarks about the property of $E_{2}^{-1,1}$ in (7.13), but they do not effect the rest of the proof at all, so the reader may skip them.

Remark 7.2. Since $\mathcal{O}(1)^{\oplus 6}$ is globally generated of rank six, a general morphism from $\mathcal{O}(-2)^{\oplus 4}$ to $\mathcal{O}(-1)^{\oplus 6}$ is a subbundle morphism, and hence its cokernel is a vector bundle. On the other hand, $E_{2}^{-1,1}$ is not a vector bundle; indeed, since $E_{2}^{-1,1}$ lies also in (7.14), we see $c_{1}\left(E_{2}^{-1,1}\right)=2$ and $c_{2}\left(E_{2}^{-1,1}\right)=7$, and if $E_{2}^{-1,1}$ were a vector bundle, it would be a nef vector bundle, so that $c_{2}\left(E_{2}^{-1,1}\right) \leq c_{1}\left(E_{2}^{-1,1}\right)^{2}=4$, which is absurd. Therefore the morphism $\mathcal{O}(-2)^{\oplus 4} \rightarrow \mathcal{O}(-1)^{\oplus 6}$ in 7.13) is not a general morphism.

Recall that (7.13) comes from the exact sequence (7.10) with $e_{0,1}=1$ :

$$
0 \rightarrow \mathcal{O}(-2)^{\oplus 2} \stackrel{\mu_{2}}{\longrightarrow} \Omega_{\mathbb{P}^{2}}(1)^{\oplus 2} \rightarrow E_{2}^{-1,1} \rightarrow 0 .
$$

Remark 7.3. We have the following commutative diagram with exact rows

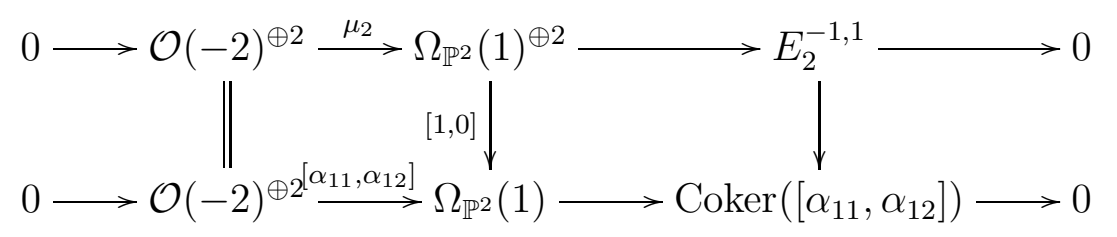

and an exact sequence

$$
0 \rightarrow \Omega_{\mathbb{P}^{2}}(1) \rightarrow E_{2}^{-1,1} \rightarrow \operatorname{Coker}\left(\left[\alpha_{11}, \alpha_{12}\right]\right) \rightarrow 0 .
$$

Since $\alpha_{11}$ and $\alpha_{12}$ are linearly independent and so are $\alpha_{11}$ and $\alpha_{21}$, there exist no elements $\lambda \in K$ and $\lambda_{i j} \in K(1 \leq i, j \leq 2)$ such that

$$
\left[\begin{array}{ll}
\alpha_{11} & \alpha_{12} \\
\alpha_{21} & \alpha_{22}
\end{array}\right]\left[\begin{array}{ll}
\lambda_{11} & \lambda_{12} \\
\lambda_{21} & \lambda_{22}
\end{array}\right]=\left[\begin{array}{l}
1 \\
\lambda
\end{array}\right]\left[\begin{array}{ll}
\alpha_{11} & \alpha_{12}
\end{array}\right]
$$

Hence no splitting injection ${ }^{t}[1, \lambda](\lambda \in K)$ of the projection $[1,0]$ induces a splitting injection of the surjection $E_{2}^{-1,1} \rightarrow \operatorname{Coker}\left(\left[\alpha_{11}, \alpha_{12}\right]\right)$.

7.2. The sheaf $E_{2}^{-1,1}$ in (7.14) is torsion-free. We shall apply the following lemma in $\$ 7.3$.

Lemma 7.9. The sheaf $E_{2}^{-1,1}$ in (7.14) is a torsion-free sheaf of rank two with $c_{1}\left(E_{2}^{-1,1}\right)=$ 2 and $c_{2}\left(E_{2}^{-1,1}\right)=7$.

Proof. Denote by $\mathcal{F}$ the cokernel of the composite of the two inclusions $\mathcal{O}(1) \rightarrow \mathcal{O}(1) \oplus$ $\mathcal{O}^{\oplus r-3}$ and $\mathcal{O}(1) \oplus \mathcal{O}^{\oplus r-3} \rightarrow \mathcal{E}$. Then $c_{1}(\mathcal{F})=2$ and $c_{2}(\mathcal{F})=7$. Moreover $\mathcal{F}$ fits in the following exact sequence

$$
0 \rightarrow \mathcal{O}^{\oplus r-3} \rightarrow \mathcal{F} \rightarrow E_{2}^{-1,1} \rightarrow 0
$$

Let $\left(e_{1}, e_{2}, \ldots, e_{r-3}\right)$ be the free basis of $\mathcal{O}^{\oplus r-3}$. For $0 \leq s \leq r-3$, denote by $\mathcal{O}^{\oplus s}$ the submodule generated by the set $\left\{e_{i} \mid i \leq s\right\}$ in $\mathcal{O}^{\oplus r-3}$ and by $\overline{\mathcal{F}}_{s}$ the quotient of $\mathcal{F}$ by $\mathcal{O}^{\oplus s}$. We have the following exact sequence

$$
0 \rightarrow \mathcal{O} \rightarrow \mathcal{F}_{s} \rightarrow \mathcal{F}_{s+1} \rightarrow 0
$$


Note that $\mathcal{F}_{0}=\mathcal{F}$ and that $\mathcal{F}_{r-3}=E_{2}^{-1,1}$.

We shall show that $\mathcal{F}_{s}$ is torsion-free for all $s(0 \leq s \leq r-3)$ by induction on $s$. Now consider the case $s=0$. Since we have (3.1) and $\mathcal{E}$ is locally free, the singular locus of $\mathcal{F}$ has codimension $\geq 2$. Hence it follows from [11, Lemma 5.4] that $\mathcal{F}$ is torsion-free; thus the claim holds for the case $s=0$.

Suppose that $\mathcal{F}_{s}$ is torsion-free. We shall show that $\mathcal{F}_{s+1}$ is torsion-free by contradiction. For simplicity, by changing the symbols, we denote $\mathcal{F}_{s}$ by $\mathcal{F}$ and $\mathcal{F}_{s+1}$ by $\mathcal{F}_{+}$, and assume that $\mathcal{F}$ is a torsoin-free sheaf with $c_{1}(\mathcal{F})=2$ and $c_{2}(\mathcal{F})=7$, that $\mathcal{F}$ can not admit a negative degree line bundle on a line or a conic as a quotient, and that $\mathcal{F}_{+}$is not torsionfree. Let $\mathcal{F}^{\vee \vee}$ be the double dual of $\mathcal{F}$. Then $\mathcal{F}^{\vee \vee}$ is a nef vector bundle with first Chern class two by Lemma 9.1. Denote by $\mathcal{Q}$ the cokernel of the inclusion $\mathcal{F} \rightarrow \mathcal{F}^{\vee \vee}$. Then we have

$$
\text { length } \mathcal{Q}=c_{2}(\mathcal{F})-c_{2}\left(\mathcal{F}^{\vee \vee}\right)=7-c_{2}\left(\mathcal{F}^{\vee \vee}\right) \text {. }
$$

Denote by $\alpha$ the composite of the two inclusions $\mathcal{O} \rightarrow \mathcal{F}$ and $\mathcal{F} \rightarrow \mathcal{F}^{\vee \vee}$, and let $\mathcal{F}^{\vee \vee} / \mathcal{O}$ be the cokernel of $\alpha$. If $h^{0}\left(\mathcal{F}^{\vee \vee}(-1)\right)=0$, then $\mathcal{F}^{\vee \vee} / \mathcal{O}$ is torsion-free by [11, Lemma 5.4]. Since $\mathcal{F}_{+1}$ is a subsheaf of $\mathcal{F}^{\vee \vee} / \mathcal{O}$ and it has a non-zero torsion subsheaf, we infer that $h^{0}\left(\mathcal{F}^{\vee \vee}(-1)\right) \neq 0$. It then follows from [11, Theorem 6.5] that $\mathcal{F}^{\vee \vee}$ satisfies one of the following:

(1) $\mathcal{F}^{\vee \vee} \cong \mathcal{O}(2) \oplus \mathcal{O}^{\oplus f-1}$

(2) $\mathcal{F}^{\vee \vee} \cong \mathcal{O}(1)^{\oplus 2} \oplus \mathcal{O}^{\oplus f-2}$

(3) $\mathcal{F}^{\vee \vee}$ fits in an exact sequence

$$
0 \rightarrow \mathcal{O}(-1) \rightarrow \mathcal{O}(1) \oplus \mathcal{O}^{\oplus f} \rightarrow \mathcal{F}^{\vee \vee} \rightarrow 0 .
$$

In particular, we see that $c_{2}\left(\mathcal{F}^{\vee \vee}\right) \leq 2$. Therefore length $\mathcal{Q} \geq 5$ by (7.21). Since $\mathcal{F}$ does not admit a negative degree line bundle on a line as a quotient, we infer that length $\left.\mathcal{Q}\right|_{L} \leq 2$ for any line $L$. Similarly we infer that length $\left.\mathcal{Q}\right|_{C} \leq 4$ for any smooth conic $C$ by the assumption on $\mathcal{F}$. If $\mathcal{Q}$ is generated by a single element, then $\mathcal{Q}$ is isomorphic to the structure sheaf $\mathcal{O}_{Z}$ of the scheme-theoretic support $Z$ of $\mathcal{Q}$. Lemma 4.4 then shows the existence of a conic $C$ such that length $Z \cap C \geq 5$. Hence length $\left.\mathcal{Q}\right|_{C}=$ length $\left.\mathcal{O}_{Z}\right|_{C} \geq 5$, which is a contradiction. In the following, we assume that $\mathcal{Q}$ is not generated by a single element.

Suppose that the case (1) holds. Let $\mathcal{Q}_{1}$ be the image of the composite of the inclusion $\mathcal{O}(2) \rightarrow \mathcal{F}^{\vee \vee}$ and the surjection $\mathcal{F}^{\vee \vee} \rightarrow \mathcal{Q}$. Denote by $\mathcal{Q}_{2}$ the quotient of $\mathcal{Q}$ by $\mathcal{Q}_{1}$. Then we have a surjection $\mathcal{O}^{\oplus f-1} \rightarrow \mathcal{Q}_{2}$. Let $\mathcal{G}$ be the kernel of this surjection $\mathcal{O}^{\oplus f-1} \rightarrow \mathcal{Q}_{2}$. Then we have a surjection $\mathcal{F} \rightarrow \mathcal{G}$. Suppose that $\mathcal{Q}_{2} \neq 0$. Then there exists a line $L$ such that length $\left.\mathcal{Q}_{2}\right|_{L} \geq 1$. This implies that the kernel of the surjection $\left.\mathcal{O}_{L}^{\oplus f-1} \rightarrow \mathcal{Q}_{2}\right|_{L}$, i.e., a quotient of $\left.\mathcal{G}\right|_{L}$ has negative degree. Since we have a surjection $\left.\left.\mathcal{F}\right|_{L} \rightarrow \mathcal{G}\right|_{L}$, this contradicts the assumption on $\mathcal{F}$. Therefore $\mathcal{Q}_{2}=0$; thus $\mathcal{Q}_{1} \cong \mathcal{Q}$. Hence we obtain a surjection $\mathcal{O}(2) \rightarrow \mathcal{Q} \cong \mathcal{Q}(2)$, and thus $\mathcal{Q}$ is generated by a single element. This contradicts the assumption. Hence the case (1) does not happen.

Suppose that we are in case (2) or (3). We have an inclusion $\mathcal{O}(1) \rightarrow \mathcal{F}^{\vee \vee}$. Denote by $\mathcal{F}^{\prime}$ the cokernel of the inclusion. If $\mathcal{F}^{\vee \vee}$ lies in the case (2), then $\mathcal{F}^{\prime} \cong \mathcal{O}(1) \oplus \mathcal{O}^{\oplus f-2}$. If $\mathcal{F}^{\vee \vee}$ lies in the case (3), then $\mathcal{F}^{\prime}$ is a torsion-free sheaf with $c_{1}\left(\mathcal{F}^{\prime}\right)=1$ and $c_{2}\left(\mathcal{F}^{\prime}\right)=1$. Hence its double dual $\mathcal{F}^{\prime \vee \vee}$ is either $\mathcal{O}(1) \oplus \mathcal{O}^{\oplus f-2}$ or $T_{\mathbb{P}^{2}}(-1) \oplus \mathcal{O}^{\oplus f-3}$. If $\mathcal{F}^{\prime \vee \vee} \cong T_{\mathbb{P}^{2}}(-1) \oplus \mathcal{O}^{\oplus f-3}$, then $c_{2}\left(\mathcal{F}^{\prime}\right)=1=c_{2}\left(\mathcal{F}^{\prime \vee V}\right)$; thus $\mathcal{F}^{\prime}$ and $\mathcal{F}^{\prime \vee \vee}$ are isomorphic. In particular $\mathcal{F}^{\prime}$ is locally free. If $\mathcal{F}^{\prime \vee \vee} \cong \mathcal{O}(1) \oplus \mathcal{O}^{\oplus f-2}$, then $\mathcal{F}^{\prime}$ is $\mathcal{I}_{q}(1) \oplus \mathcal{O}^{\oplus f-2}$ for some point $q$. Let $\mathcal{H}_{1}$ and $\mathcal{Q}_{1}$ 
be respectively the kernel and the image of the composite of the inclusion $\mathcal{O}(1) \rightarrow \mathcal{F}^{\vee \vee}$ and the projection $\mathcal{F}^{\vee \vee} \rightarrow \mathcal{Q}$. Denote by $\mathcal{G}$ the quotient of $\mathcal{F}$ by $\mathcal{H}_{1}$ and by $\mathcal{Q}_{2}$ the quotient of $\mathcal{Q}$ by $\mathcal{Q}_{1}$. Note that $\mathcal{H}_{1} \cong \mathcal{I}_{Z_{1}}(1)$ for some 0 -dimensional closed subscheme $Z_{1}$ and that $\mathcal{Q}_{1} \cong \mathcal{O}_{Z_{1}}$. Then we have the following commutative diagram with exact rows and columns.

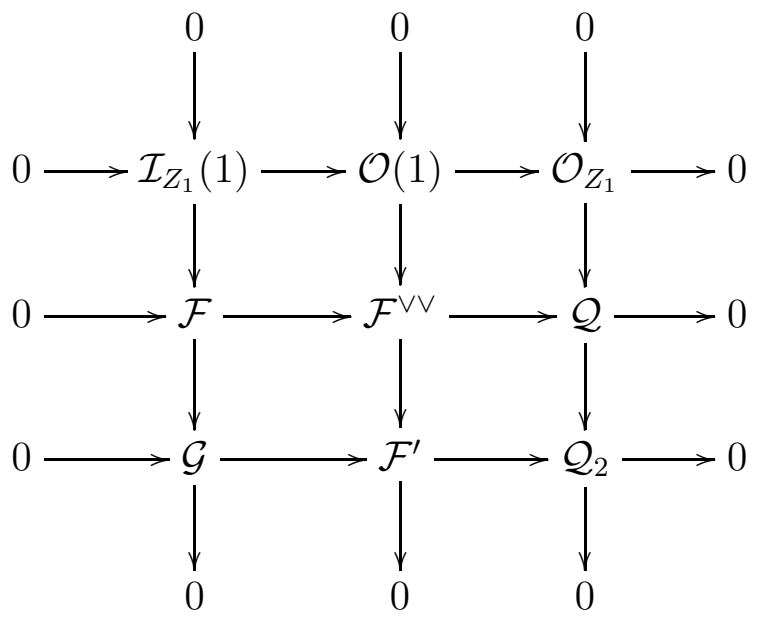

We claim that length $\left.\mathcal{Q}_{2}\right|_{L} \leq 1$ for any line $L$; this follows from the fact that $\left.\mathcal{G}\right|_{L}$ can not admit a line bundle of negative degree as a quotient, but a careful argument is needed if $\mathcal{F}^{\prime} \cong \mathcal{I}_{q}(1) \oplus \mathcal{O}^{\oplus f-1}$ and $q \in L$; suppose that length $\left.\mathcal{Q}_{2}\right|_{L} \geq 1$ for a line $L$ passing through $q$. Note here that $\left.\mathcal{I}_{q}(1)\right|_{L}$ is isomorphic to $\mathcal{O}_{L} \oplus k(q)$. Since $\left.\mathcal{G}\right|_{L}$ does not admit a line bundle of negative degree as a quotient, we infer that the composite of the inclusion $\left.\left.k(q) \rightarrow \mathcal{I}_{q}(1)\right|_{L} \rightarrow \mathcal{F}^{\prime}\right|_{L}$ and the surjection $\left.\left.\mathcal{F}^{\prime}\right|_{L} \rightarrow \mathcal{Q}_{2}\right|_{L}$ must be surjective. Hence length $\left.\mathcal{Q}_{2}\right|_{L}=1$. Therefore the claim holds,

The claim above implies, in particular, that the number of minimal (non-zero) generators of $\mathcal{Q}_{2, p}$ at each point $p$ is at most one. Hence it follows from the claim above that $\mathcal{Q}_{2}$ is isomorphic to the residue field $k(p)$ of some point $p$ unless $\mathcal{Q}_{2}$ is zero.

If $\mathcal{Q}_{2}=0$, then $\mathcal{Q} \cong \mathcal{Q}_{1} \cong \mathcal{O}_{Z_{1}}$, and this case does not happen by our assumption. Suppose that $\mathcal{Q}_{2} \cong k(p)$. Then length $Z_{1} \geq 4$. If $p \notin Z_{1}$, then the support $Z$ of $\mathcal{Q}$ is the disjoint union of $Z_{1}$ and $p$, and we see that $\mathcal{Q} \cong \mathcal{O}_{Z}$; this case is also ruled out. In the following, we assume that $p \in Z_{1}$.

We claim that length $Z_{1} \cap L \leq 2$ for any line $L$. If $\left.\left.\mathcal{O}_{Z_{1}}\right|_{L} \rightarrow \mathcal{Q}\right|_{L}$ is injective, then the claim holds since length $\left.\mathcal{Q}\right|_{L} \leq 2$. Suppose that $\left.\left.\mathcal{O}_{Z_{1}}\right|_{L} \rightarrow \mathcal{Q}\right|_{L}$ is not injective. Then $p \in L$. Since $\left.\mathcal{O}_{L}(1) \rightarrow \mathcal{F}^{\vee \vee}\right|_{L}$ is injective, we have the following exact sequence

$$
\left.\left.\kappa \rightarrow \mathcal{O}_{Z_{1}}\right|_{L} \rightarrow \mathcal{Q}\right|_{L} \rightarrow k(p) \rightarrow 0,
$$

where $\kappa$ denotes the kernel of the morphism $\left.\left.\mathcal{G}\right|_{L} \rightarrow \mathcal{F}^{\prime}\right|_{L}$. Since we have the following commutative diagram with exact rows,

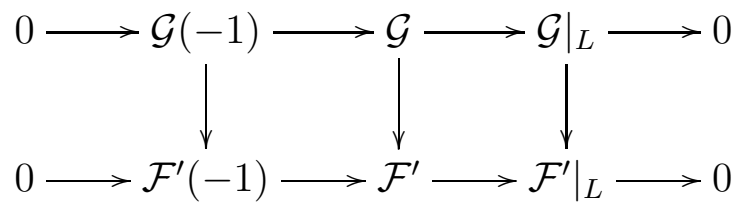

we see that $\kappa \cong k(p)$. Since $\left.\left.\mathcal{O}_{Z_{1}}\right|_{L} \rightarrow \mathcal{Q}\right|_{L}$ is not injective, this implies that there exists the following exact sequence

$$
\left.\left.0 \rightarrow k(p) \rightarrow \mathcal{O}_{Z_{1}}\right|_{L} \rightarrow \mathcal{Q}\right|_{L} \rightarrow k(p) \rightarrow 0
$$


Therefore length $Z_{1} \cap L=$ length $\left.\mathcal{O}_{Z_{1}}\right|_{L}=$ length $\left.\mathcal{Q}\right|_{L} \leq 2$.

We claim here that the torsion subsheaf of $\mathcal{F}^{\vee \vee} / \mathcal{O}$ is isomorphic to $\mathcal{O}_{L}(1)$ for some line $L$ containing $p$. First note that if $\alpha$ factors through some subsheaf $\mathcal{O}(1)$ then the claim holds. Suppose that $\mathcal{F}^{\vee \vee} \cong \mathcal{O}(1)^{\oplus 2} \oplus \mathcal{O}^{\oplus f-2}$. If $\alpha$ does not factor through $\mathcal{O}(1)^{\oplus 2}$, then $\mathcal{F}^{\vee \vee} / \mathcal{O}$ becomes locally free. This contradicts that $\mathcal{F}_{+}$has a non-zero tosion subsheaf. Therefore $\alpha$ factors through $\mathcal{O}(1)^{\oplus 2}$. Since $\mathcal{F}^{\vee \vee} / \mathcal{O}$ can not be torsion-free, we see that the induced morphism $\mathcal{O} \rightarrow \mathcal{O}(1)^{\oplus 2}$ factors through a direct summand $\mathcal{O}(1)$. Therefore the claim holds in this case. Suppose that we are in case (3). Then $\mathcal{F}^{\prime}$ is either $T_{\mathbb{P}^{2}}(-1) \oplus \mathcal{O}^{\oplus f-3}$ or $\mathcal{I}_{p}(1) \oplus \mathcal{O}^{\oplus f-2}$. If $\alpha$ does not factor through $\mathcal{O}(1)$, then we have an injection $\mathcal{O} \rightarrow \mathcal{F}^{\prime}$. If $\mathcal{F}^{\prime}=T_{\mathbb{P}^{2}}(-1) \oplus \mathcal{O}^{\oplus f-3}$, then it follows from [11, Lemma 5.4] that $\mathcal{F}^{\prime} / \mathcal{O}$ is torsion-free; thus $\mathcal{F}^{\vee \vee} / \mathcal{O}$ is torsion-free. This is a contradiction. If $\mathcal{F}^{\prime}=\mathcal{I}_{p}(1) \oplus \mathcal{O}^{\oplus f-2}$, the cokernel of the morphism $\mathcal{O} \rightarrow \mathcal{F}^{\prime}$ is isomorphic to $\mathcal{O}_{L} \oplus \mathcal{O}^{\oplus f-2}$ for some line $L$ containing $p$, since $\mathcal{F}^{\vee \vee} / \mathcal{O}$ is not torsion-free. Now we have the following commutative diagram with exact rows.

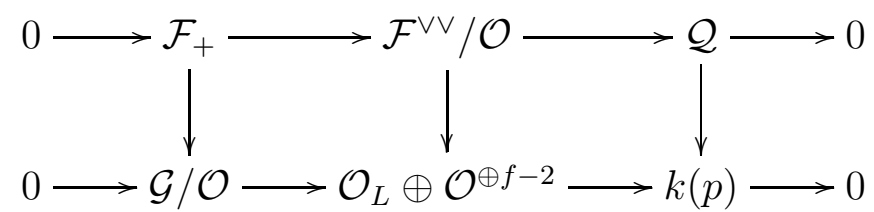

We see that $\left.(\mathcal{G} / \mathcal{O})\right|_{L}$ admits a negative degree line bundle as a quotient. This is a contradiction. Therefore $\alpha$ factors through the subsheaf $\mathcal{O}(1)$ and the claim also holds in this case.

The claim above implies that we have the following commutative diagram with exact rows and columns

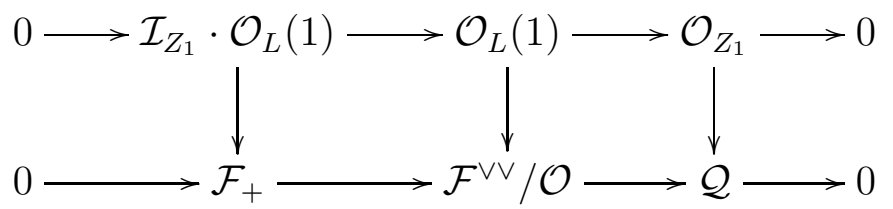

where $\mathcal{I}_{Z_{1}} \cdot \mathcal{O}_{L}$ denotes the inverse image ideal sheaf. In particular, we infer that $Z_{1}$ lies on the line $L$. Since length $Z_{1} \geq 4$, this contradicts the claim that length $Z_{1} \cap L \leq 2$. Therefore we conclude that $\mathcal{F}_{+}$is torsion-free.

7.3. Proof for the case $n=2$ and $h^{1}(\mathcal{E})>0$. Set $s=h^{1}(\mathcal{E})$. Then we have the following exact sequence

$$
0 \rightarrow \mathcal{E} \rightarrow \mathcal{E}_{0} \rightarrow \mathcal{O}^{\oplus s} \rightarrow 0
$$

which induces an isomorphism $H^{0}\left(\mathcal{O}^{\oplus s}\right) \cong H^{1}(\mathcal{E})$. It follows from [10, Theorem 6.2.12 (ii)] that $\mathcal{E}_{0}$ is a nef vector bundle of rank $r+s$ with first Chern class three, second Chern class nine, and $h^{1}\left(\mathcal{E}_{0}\right)=0$. Since $H^{0}(\mathcal{E}) \cong H^{0}\left(\mathcal{E}_{0}\right)$, the image $\mathcal{E}_{1}$ of the evaluation map $H^{0}\left(\mathcal{E}_{0}\right) \otimes \mathcal{O} \rightarrow \mathcal{E}_{0}$ is contained in $\mathcal{E}$. Denote by $\mathcal{F}$ the quotient of $\mathcal{E}$ by $\mathcal{E}_{1}$. By abuse of notation, we also denote by $E_{2}^{-1,1}$ the cokernel of the evaluation map $H^{0}\left(\mathcal{E}_{0}\right) \otimes \mathcal{O} \rightarrow \mathcal{E}_{0}$. Then we have the following commutative diagram with exact rows.

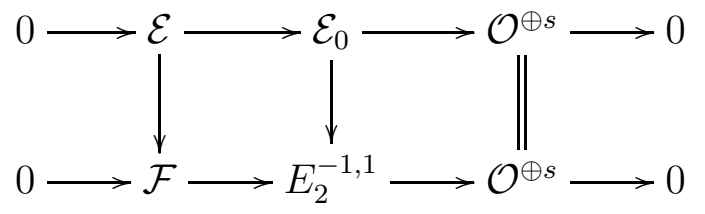


Since we have a surjection $E_{2}^{-1,1} \rightarrow \mathcal{O}^{\oplus s}$ with $s \geq 1$, Remark 7.1 and Lemmas 7.8 and 7.9 imply that $E_{2}^{-1,1}$ is either $\mathcal{O}$ or a torsion-free sheaf of rank two with $c_{1}\left(E_{2}^{-1,1}\right)=2$ and $c_{2}\left(E_{2}^{-1,1}\right)=7$. If $E_{2}^{-1.1}$ is the latter, then $s=1$ and $\mathcal{F} \cong \mathcal{I}_{Z}(2)$ for some 0-dimensional closed subscheme $Z$ of length seven. Lemma 4.4 then shows that $\mathcal{E}$ admits a negative degree quotient, which contradicts that $\mathcal{E}$ is nef. Hence $E_{2}^{-1,1} \cong \mathcal{O}$, and thus $\mathcal{F}=0$, $\mathcal{E}_{1}=\mathcal{E}$, and $\mathcal{E}_{0}$ and $\mathcal{E}_{1}$ fit in the following exact sequences

$$
\begin{aligned}
0 \rightarrow \mathcal{O}(-3) \rightarrow \mathcal{O}^{\oplus r+1} \rightarrow \mathcal{E}_{1} \rightarrow 0 ; \\
0 \rightarrow \mathcal{E}_{1} \rightarrow \mathcal{E}_{0} \rightarrow \mathcal{O} \rightarrow 0
\end{aligned}
$$

as in (7.17) and (7.18). Hence $\mathcal{E}$ fits in an exact sequence

$$
0 \rightarrow \mathcal{O}(-3) \rightarrow \mathcal{O}^{\oplus r+1} \rightarrow \mathcal{E} \rightarrow 0 .
$$

This is the case (15) of Theorem 1, where $n=2$.

7.4. Proof for the case $n \geq 3$. What we have to show in case $n \geq 3$ is the following lemma.

Lemma 7.10. If $n \geq 3$, then $c_{3}=27$ and $h^{0}(\mathcal{E}(-1)) \leq 1$. Moreover $\mathcal{E}$ satisfies one of the following:

(1) $n \geq 3, h^{0}(\mathcal{E}(-1))=0, h^{n-3}(\mathcal{E}(2-n))=0$, and $\mathcal{E}$ lies in the case (15) of Theorem 1 ;

(2) $n \geq 3$ and $h^{0}(\mathcal{E}(-1))=1$;

(3) $n \geq 4, h^{0}(\mathcal{E}(-1))=0$, and $h^{n-3}(\mathcal{E}(2-n))=1$.

Proof. We shall first show that $h^{0}(\mathcal{E}(-1)) \leq 1$ by induction on $n \geq 3$. It follows from (3.1) that $h^{0}(\mathcal{E}(-1)) \leq h^{0}\left(\left.\mathcal{E}\right|_{H}(-1)\right)$ for any hyperplane $H$ in $\mathbb{P}^{n}$. Note here that $h^{0}\left(\left.\mathcal{E}\right|_{H}(-1)\right) \leq$ 1 by induction hypothesis if $n \geq 4$ and by what we have seen in $\$ 7.1$ and $\$ 7.3$ if $n=3$. Hence $h^{0}(\mathcal{E}(-1)) \leq 1$.

Recall here that $\mathcal{E}$ is globally generated if $h^{1}(\mathcal{E}(-1))=0$ and $\left.\mathcal{E}\right|_{H}$ is globally generated for any hyperplane $H$ in $\mathbb{P}^{n}$ by [15, Lemma 3]. In order to show that $\mathcal{E}$ lies in the case (15) of Theorem 1, it is enough to show that $h^{0}(\mathcal{E})=r+1$ and that $\mathcal{E}$ is globally generated.

Suppose that $n=3$. As we have seen in $\$ 7.3$, we have $h^{1}\left(\left.\mathcal{E}\right|_{H}\right) \leq 1$.

Suppose furthermore that $h^{0}(\mathcal{E}(-1))=1$. Then we get the case $(2)$ of Lemma 7.10 in case $n=3$. Note here that we have $c_{3}=27$ in this case. Indeed, the argument above shows that $h^{0}\left(\left.\mathcal{E}\right|_{H}(-1)\right)=1$, and this implies that $h^{1}\left(\left.\mathcal{E}\right|_{H}\right)=0$ as we have also seen in $\$ 7.1$ and $\$ 7.3$. It then follows from (3.2) and (3.4) that $h^{q}(\mathcal{E}(-1))=0$ for $q \geq 2$. Now we have

$$
1 \geq 1-h^{1}(\mathcal{E}(-1))=\chi(\mathcal{E}(-1))=\left(c_{3}-25\right) / 2
$$

by (3.20). Note here that $c_{3} \geq 27$ by (3.23). Hence we infer that $c_{3}=27$ and that $h^{1}(\mathcal{E}(-1))=0$.

Suppose furthermore that $h^{0}(\mathcal{E}(-1))=0$. It follows from $h^{1}\left(\left.\mathcal{E}\right|_{H}\right) \leq 1$ and (3.2) that $h^{2}(\mathcal{E}(-1)) \leq 1$ and that equality holds if and only if $h^{1}\left(\left.\mathcal{E}\right|_{H}\right)=1$. Moreover we have $h^{3}(\mathcal{E}(-1))=0$ by (3.2) and (3.4). It then follows from (3.20) that

$$
1 \geq-h^{1}(\mathcal{E}(-1))+h^{2}(\mathcal{E}(-1))=\chi(\mathcal{E}(-1))=\left(c_{3}-25\right) / 2
$$

Since $c_{3} \geq 27$ by (3.23), this implies that $c_{3}=27$, that $h^{2}(\mathcal{E}(-1))=1$, and that $h^{1}(\mathcal{E}(-1))=0$. Hence $h^{1}\left(\left.\mathcal{E}\right|_{H}\right)=1$. As we have seen in 97.3 , this implies that $\left.\mathcal{E}\right|_{H}$ is globally generated and that $h^{0}\left(\left.\mathcal{E}\right|_{H}\right)=r+1$. Therefore $\mathcal{E}$ is globally generated and $h^{0}(\mathcal{E})=r+1$. This is the case (1) of Lemma 7.10 in case $n=3$. 
Suppose that $n \geq 4$. If $h^{0}(\mathcal{E}(-1))=1$, we get the case (2) of Lemma 7.10. Suppose that $h^{0}(\mathcal{E}(-1))=0$. We shall show that $h^{n-3}(\mathcal{E}(2-n)) \leq 1$ by induction on $n \geq 4$. Note here that $h^{q}(\mathcal{E}(3-n))=0$ for all $q>0$ by (3.2) . Hence $h^{n-3}(\mathcal{E}(2-n)) \leq h^{n-4}\left(\left.\mathcal{E}\right|_{H}(3-n)\right) \leq 1$ by induction hypothesis if $n \geq 5$ and by what we have shown, i.e., $h^{0}\left(\left.\mathcal{E}\right|_{H}(-1)\right) \leq 1$ if $n=4$. If $h^{n-3}(\mathcal{E}(2-n))=1$, we obtain the case (3) of Lemma 7.10.

Suppose that $n \geq 4$, that $h^{0}(\mathcal{E}(-1))=0$, and that $h^{n-3}(\mathcal{E}(2-n))=0$. We claim here that $\mathcal{E}$ lies in the case $(15)$ of Theorem 1. We proceed by induction not only on $n \geq 4$ but also on $n \geq 3$; if $n=3$, then the two conditions $h^{0}(\mathcal{E}(-1))=0$ and $h^{n-3}(\mathcal{E}(2-n))=0$ become the same and $\mathcal{E}$ lies in the case $(15)$ of Theorem 1 as we have seen above. Suppose now that $n \geq 4$. The assumption $h^{n-3}(\mathcal{E}(2-n))=0$ implies that $h^{n-4}\left(\left.\mathcal{E}\right|_{H}(3-n)\right)=0$ by (3.2) if $n \geq 5$ and by the assumption $h^{0}(\mathcal{E}(-1))=0$ if $n=4$. Hence we infer that $h^{0}\left(\left.\mathcal{E}\right|_{L^{3}}(-1)\right)=h^{n-3}(\mathcal{E}(2-n))=0$ for any linear subspace $L^{3}$ of dimension three in $\mathbb{P}^{n}$. Therefore we see that $h^{0}\left(\left.\mathcal{E}\right|_{H}(-1)\right) \leq h^{0}\left(\left.\mathcal{E}\right|_{L^{3}}(-1)\right)=0$ by (3.1). Now it follows from the induction hypothesis that $\left.\mathcal{E}\right|_{H}$ lies in the case (15) of Theorem 1. Thus $h^{0}\left(\left.\mathcal{E}\right|_{H}\right)=r+1$ and $\left.\mathcal{E}\right|_{H}$ is globally generated. Moreover $h^{1}(\mathcal{E}(-1))=0$ by $(3.2)$ and $h^{0}(\mathcal{E}(-1))=0$ by assumption. Hence $h^{0}(\mathcal{E})=r+1$ and $\mathcal{E}$ is globally generated.

\section{Several Remarks on Theorem 1}

Remark 8.1. The exact sequence in the case (7) of Theorem 1 induces the following

$$
0 \rightarrow T_{\mathbb{P}^{3}}(-2) \rightarrow \mathcal{O}(1) \oplus \mathcal{O}^{\oplus r+2} \rightarrow \mathcal{E} \rightarrow 0,
$$

and, dualizing this, we obtain the following exact sequence

$$
0 \rightarrow \mathcal{E}^{\vee} \rightarrow \mathcal{O}(-1) \oplus \mathcal{O}^{\oplus r+2} \rightarrow \Omega_{\mathbb{P}^{3}}(2) \rightarrow 0 .
$$

Note that the injection $H^{0}\left(\mathcal{E}^{\vee}\right) \rightarrow H^{0}\left(\mathcal{O}(-1) \oplus \mathcal{O}^{\oplus r+2}\right)$ induces a splitting injection $\mathcal{O} \otimes$ $H^{0}\left(\mathcal{E}^{\vee}\right) \rightarrow \mathcal{O}^{\oplus r+2}$ and that the composite of two splitting injection $\mathcal{O} \otimes H^{0}\left(\mathcal{E}^{\vee}\right) \rightarrow \mathcal{O}^{\oplus r+2}$ and $\mathcal{O}^{\oplus r+2} \rightarrow \mathcal{O}(-1) \oplus \mathcal{O}^{\oplus r+2}$ is equal to the composite of $\mathcal{O} \otimes H^{0}\left(\mathcal{E}^{\vee}\right) \rightarrow \mathcal{E}^{\vee}$ and $\mathcal{E}^{\vee} \rightarrow$ $\mathcal{O}(-1) \oplus \mathcal{O}^{\oplus r+2}$. Thus $\mathcal{O} \otimes H^{0}\left(\mathcal{E}^{\vee}\right) \rightarrow \mathcal{E}^{\vee}$ is also a splitting injection. Hence $\mathcal{E}^{\vee} \cong$ $\mathcal{E}_{0}^{\vee} \oplus \mathcal{O} \otimes H^{0}\left(\mathcal{E}^{\vee}\right)$ for some vector bundle $\mathcal{E}_{0}$ of rank $s=r-h^{0}\left(\mathcal{E}^{\vee}\right)$. Since $c_{3}\left(\mathcal{E}_{0}\right)=2 \neq 0$, we infer that $s \geq 3$. Note that $h^{0}\left(\mathcal{E}_{0}^{\vee}\right)=0$ and that $\mathcal{E}_{0}^{\vee}$ fits in an exact sequence

$$
0 \rightarrow \mathcal{E}_{0}^{\vee} \rightarrow \mathcal{O}(-1) \oplus \mathcal{O}^{\oplus s+2} \rightarrow \Omega_{\mathbb{P}^{3}}(2) \rightarrow 0 .
$$

Since $h^{0}\left(\Omega_{\mathbb{P}^{3}}(2)\right)=6$ by the Bott formula [14, p. 8], we see that $s \leq 4$. Moreover $h^{1}\left(\mathcal{E}_{0}^{\vee}\right)=$ $4-s$. The image of $\mathcal{E}_{0}^{\vee} \rightarrow \mathcal{O}(-1) \oplus \mathcal{O}^{\oplus s+2} \rightarrow \mathcal{O}(-1)$ is $\mathcal{I}_{Z}(-1)$ for some closed subscheme $Z$ in $\mathbb{P}^{3}$.

Suppose that $Z=\emptyset$. Let $\mathcal{F}^{\vee}$ be the kernel of the surjection $\mathcal{E}_{0}^{\vee} \rightarrow \mathcal{O}(-1)$. Then $\mathcal{F}$ fits in an exact sequence

$$
0 \rightarrow T_{\mathbb{P}^{3}}(-2) \rightarrow \mathcal{O}^{\oplus s+2} \rightarrow \mathcal{F} \rightarrow 0
$$

and $\mathcal{F}$ is a nef vector bundle of rank $s-1$ with $c_{1}(\mathcal{F})=2$. Moreover, as we have seen in [11, Remark 6.7], $\mathcal{F} \cong \Omega_{\mathbb{P}^{3}}(2)$ if $s=4$ and $\mathcal{F} \cong \mathcal{N}(1)$ if $s=3$ where $\mathcal{N}$ is a null correlation bundle on $\mathbb{P}^{3}$. Hence $\mathcal{E}$ is either $\mathcal{O}(1) \oplus \Omega_{\mathbb{P}^{3}}(2) \oplus \mathcal{O}^{\oplus r-4}$ or $\mathcal{O}(1) \oplus \mathcal{N}(1) \oplus \mathcal{O}^{\oplus r-3}$ if $Z=\emptyset$.

Remark 8.2. Suppose that $\mathcal{E}$ is in the case (8) of Theorem 1. Then $\mathcal{E}$ has $\mathcal{O}^{\oplus r-4}$ as a subbundle; let $\mathcal{E}_{0}$ be the quotient bundle $\mathcal{E} / \mathcal{O}^{\oplus r-4}$ of rank four. In [1, §6 III (a)], it is stated that $\mathcal{E}_{0} \cong \Omega_{\mathbb{P}^{4}}(2)$. (Therefore we see that $\mathcal{E} \cong \Omega_{\mathbb{P}^{4}}(2) \oplus \mathcal{O}^{\oplus r-4}$.) 
For the sake of completeness, we give a different proof of this result in our context. First note that $\mathcal{E}_{0}$ fits in an exact sequence

$$
0 \rightarrow T_{\mathbb{P}^{4}}(-3) \rightarrow \mathcal{O}(-1)^{\oplus 10} \rightarrow \mathcal{O}^{\oplus 10} \rightarrow \mathcal{E}_{0} \rightarrow 0 .
$$

Therefore we obtain the following exact sequence

$$
0 \rightarrow \mathcal{E}_{0}^{\vee}(-1) \rightarrow \mathcal{O}(-1)^{\oplus 10} \rightarrow \mathcal{O}^{\oplus 10} \rightarrow \Omega_{\mathbb{P}^{4}}(2) \rightarrow 0 .
$$

We split this sequence into the following two exact sequences:

$$
\begin{aligned}
0 \rightarrow \mathcal{E}_{0}^{\vee}(-1) \rightarrow \mathcal{O}(-1)^{\oplus 10} \rightarrow \mathcal{G} \rightarrow 0 ; \\
0 \rightarrow \mathcal{G} \rightarrow \mathcal{O}^{\oplus 10} \rightarrow \Omega_{\mathbb{P}^{4}}(2) \rightarrow 0 .
\end{aligned}
$$

We claim here that the induced map $H^{0}\left(\mathcal{O}^{\oplus 10}\right) \rightarrow H^{0}\left(\Omega_{\mathbb{P}^{4}}(2)\right)$ is an isomorphism. Since $h^{0}\left(\Omega_{\mathbb{P}^{4}}(2)\right)=10$ by the Bott formula [14, p. 8], it is enough to show that the map is injective. Suppose, to the contrary, that there exists a subbundle $\mathcal{O} \rightarrow \mathcal{O}^{\oplus 10}$ such that the composite $\mathcal{O} \rightarrow \mathcal{O}^{\oplus 10} \rightarrow \Omega_{\mathbb{P}^{4}}(2)$ is zero. Then the subbundle morphism $\mathcal{O} \rightarrow \mathcal{O}^{\oplus 10}$ induces a subbundle morphism $\mathcal{O} \rightarrow \mathcal{G}$; let $\mathcal{G}_{0}$ be the quotient bundle $\mathcal{G} / \mathcal{O}$. The composite of the subbundle morphism $\mathcal{O} \rightarrow \mathcal{G}$ and the extension class in $\operatorname{Ext}^{1}\left(\mathcal{G}, \mathcal{E}_{0}^{\vee}(-1)\right)$ corresponding to (8.1) lies in $H^{1}\left(\mathcal{E}_{0}^{\vee}(-1)\right)$, and it gives rise to an exact sequence

$$
0 \rightarrow \mathcal{E}_{0}^{\vee} \rightarrow \mathcal{F} \rightarrow \mathcal{O}(1) \rightarrow 0 .
$$

Then $\mathcal{F}$ is a vector bundle, and it also fits in an exact sequence

$$
0 \rightarrow \mathcal{F} \rightarrow \mathcal{O}^{\oplus 10} \rightarrow \mathcal{G}_{0}(1) \rightarrow 0 .
$$

Hence $\mathcal{F}^{\vee}$ is nef. On the other hand, it follows from (8.3) that $c_{3}\left(\mathcal{F}^{\vee}\right)=-2$ since $c_{2}=4$ and $c_{3}=2$. This contradicts the non-negativity of the Chern classes of nef vector bundles. Therefore the claim holds. Hence we may assume that the dual of (8.2) is nothing but the exact sequence induced by the two wedge $\wedge^{2}\left(\mathcal{O}^{\oplus 5}\right) \rightarrow \wedge^{2}\left(T_{\mathbb{P}^{4}}(-1)\right)$ of the surjection in the Euler exact sequence

$$
0 \rightarrow \mathcal{O}(-1) \rightarrow \mathcal{O}^{\oplus 5} \rightarrow T_{\mathbb{P}^{4}}(-1) \rightarrow 0 .
$$

In particular, $\mathcal{G} \cong \Omega_{\mathbb{P}^{4}}^{2}(2)$. Thus the exact sequence (8.1) implies an exact sequence

$$
0 \rightarrow \mathcal{E}_{0}^{\vee} \rightarrow \mathcal{O}^{\oplus 10} \rightarrow \Omega_{\mathbb{P}^{4}}^{2}(3) \rightarrow 0 .
$$

Next we claim that the induced map $H^{0}\left(\mathcal{O}^{\oplus 10}\right) \rightarrow H^{0}\left(\Omega_{\mathbb{P}^{4}}^{2}(3)\right)$ is an isomorphism. Since $h^{0}\left(\Omega_{\mathbb{P}^{4}}^{2}(3)\right)=10$ by the Bott formula, it is enough to show that the map is injective. Suppose, to the contrary, that there exists a subbundle $\mathcal{O} \rightarrow \mathcal{O}^{\oplus 10}$ such that the composite $\mathcal{O} \rightarrow \mathcal{O}^{\oplus 10} \rightarrow \Omega_{\mathbb{P}^{4}}^{2}(3)$ is zero. Then the subbundle morphism $\mathcal{O} \rightarrow \mathcal{O}^{\oplus 10}$ induces a subbundle morphism $\mathcal{O} \rightarrow \mathcal{E}_{0}^{\vee}$; let $\mathcal{E}_{1}^{\vee}$ be the quotient bundle $\mathcal{E}_{0}^{\vee} / \mathcal{O}$. Then $\mathcal{E}_{1}^{\vee}$ fits in an exact sequence

$$
0 \rightarrow \mathcal{E}_{1}^{\vee} \rightarrow \mathcal{O}^{\oplus 9} \rightarrow \Omega_{\mathbb{P}^{4}}^{2}(3) \rightarrow 0 .
$$

Hence it follows from the Bott formula that $h^{0}\left(\mathcal{E}_{1}\right)=9$. Since $h^{0}\left(\mathcal{E}_{0}\right)=10$, this implies that $\mathcal{E}_{0} \cong \mathcal{E}_{1} \oplus \mathcal{O}$. Thus $c_{4}\left(\mathcal{E}_{0}\right)=0$, which however contradicts that $c_{4}=1$. Therefore $H^{0}\left(\mathcal{O}^{\oplus 10}\right) \rightarrow H^{0}\left(\Omega_{\mathbb{P}^{4}}^{2}(3)\right)$ is an isomorphism, and we conclude that the exact sequence (8.5) is nothing but the exact sequence induced by the two wedge $\wedge^{2}\left(\mathcal{O}^{\oplus 5}\right) \rightarrow \wedge^{2}\left(T_{\mathbb{P}^{4}}(-1)\right)$ of the surjection in the Euler exact sequence (8.4). Therefore $\mathcal{E}_{0} \cong \Omega_{\mathbb{P}^{4}}(2)$. 
Remark 8.3. Suppose that $n=4$ and that $\mathcal{E}$ fits in an exact sequence in the case (10) of Theorem 1 . Then $\mathcal{E}$ is an extension of the Tango bundle by a trivial bundle $\mathcal{O}^{\oplus r-3}$.

The reason is as follows. Since $\mathcal{E}$ is globally generated, $\mathcal{E}$ has $\mathcal{O}^{\oplus r-4}$ as a subbundle; denote by $\mathcal{E}_{0}$ the quotient bundle $\mathcal{E} / \mathcal{O}^{\oplus r-4}$. Since $\mathcal{E}_{0}$ is globally generated of rank four with $c_{4}\left(\mathcal{E}_{0}\right)=0, \mathcal{E}_{0}$ has also $\mathcal{O}$ as a subbundle; denote by $\mathcal{E}_{1}$ the quotient bundle $\mathcal{E}_{0} / \mathcal{O}$. We show that $\mathcal{E}_{1}$ is the Tango bundle. First note that the dual $\mathcal{E}_{1}^{\vee}$ of $\mathcal{E}_{1}$ fits in an exact sequence

$$
0 \rightarrow \mathcal{E}_{1}^{\vee} \rightarrow \mathcal{O}^{\oplus 7} \rightarrow \Omega_{\mathbb{P}^{4}}(2) \rightarrow 0 .
$$

Note also that $h^{0}\left(\mathcal{E}_{1}^{\vee}\right)=0$; indeed, if $h^{0}\left(\mathcal{E}_{1}^{\vee}\right) \neq 0$, then $\mathcal{E}_{1}$ would admit $\mathcal{O}$ as a direct summand, which contradicts the fact that $c_{3}\left(\mathcal{E}_{1}\right)=5 \neq 0$ and the rank of $\mathcal{E}_{1}$ is three. Since $h^{0}\left(\Omega_{\mathbb{P}^{4}}(2)\right)=10$ by the Bott formula [14, p. 8], this implies that $h^{1}\left(\mathcal{E}_{1}^{\vee}\right)=3$. Now we have an isomorphism $\operatorname{Ext}^{1}\left(\operatorname{Ext}^{1}\left(\mathcal{O}, \mathcal{E}_{1}^{\vee}\right) \otimes \mathcal{O}, \mathcal{E}_{1}^{\vee}\right) \cong \operatorname{End}\left(\operatorname{Ext}^{1}\left(\mathcal{O}, \mathcal{E}_{1}^{\vee}\right)\right)$; let $\xi$ be the element in $\operatorname{Ext}^{1}\left(\operatorname{Ext}^{1}\left(\mathcal{O}, \mathcal{E}_{1}^{\vee}\right) \otimes \mathcal{O}, \mathcal{E}_{1}^{\vee}\right)$ corresponding to the identity in $\operatorname{End}\left(\operatorname{Ext}^{1}\left(\mathcal{O}, \mathcal{E}_{1}^{\vee}\right)\right)$. Consider the extension

$$
0 \rightarrow \mathcal{E}_{1}^{\vee} \rightarrow \mathcal{F} \rightarrow \operatorname{Ext}^{1}\left(\mathcal{O}, \mathcal{E}_{1}^{\vee}\right) \otimes \mathcal{O} \rightarrow 0
$$

corresponding to $\xi$; then $H^{0}(\mathcal{F}) \cong H^{0}\left(\mathcal{E}_{1}^{\vee}\right)=0$ and $H^{1}(\mathcal{F})=0$. Let

$$
0 \rightarrow \mathcal{O}^{\oplus 7} \rightarrow \mathcal{O}^{\oplus 10} \rightarrow \operatorname{Ext}^{1}\left(\mathcal{O}, \mathcal{E}_{1}^{\vee}\right) \otimes \mathcal{O} \rightarrow 0
$$

be the extension corresponding to the image of $\xi$ via the map

$$
\operatorname{Ext}^{1}\left(\operatorname{Ext}^{1}\left(\mathcal{O}, \mathcal{E}_{1}^{\vee}\right) \otimes \mathcal{O}, \mathcal{E}_{1}^{\vee}\right) \rightarrow \operatorname{Ext}^{1}\left(\operatorname{Ext}^{1}\left(\mathcal{O}, \mathcal{E}_{1}^{\vee}\right) \otimes \mathcal{O}, \mathcal{O}^{\oplus 7}\right) .
$$

Then $\mathcal{F}$ fits in an exact sequence

$$
0 \rightarrow \mathcal{F} \rightarrow \mathcal{O}^{\oplus 10} \rightarrow \Omega_{\mathbb{P}^{4}}(2) \rightarrow 0 .
$$

Since $h^{0}(\mathcal{F})=h^{1}(\mathcal{F})=0$, the induced map $H^{0}\left(\mathcal{O}^{\oplus 10}\right) \rightarrow H^{0}\left(\Omega_{\mathbb{P}^{4}}(2)\right)$ is an isomorphism. Therefore $\mathcal{F}^{\vee} \cong \Omega_{\mathbb{P}^{4}}^{2}(3)$, and thus $\mathcal{E}_{1}$ is the Tango bundle.

According to [8, \$4], Trautmann [20] and Vetter [21] give an explicit construction of the bundle which is, up to taking duals and twists by $\mathcal{O}(1)$, the Tango bundle.

In the following, we give an example in case (16) of Theorem 1.

Example 1. Let $\psi: \mathcal{O}(-2)^{\oplus 3} \rightarrow \mathcal{O}(-1)^{\oplus 3}$ be the morphism defined by a matrix

$$
\left[\begin{array}{ccc}
0 & y & x-\lambda z \\
x & 0 & y \\
z & z-x & 0
\end{array}\right],
$$

where $(x: y: z)$ are homogeneous coordinates of $\mathbb{P}^{2}$ and $\lambda \in K \backslash\{0,1\}$. Then $\operatorname{Coker}(\psi)$ is supported on an elliptic curve $E: y^{2} z=x(x-z)(x-\lambda z)$, and its Chern polynomial $c_{t}(\operatorname{Coker}(\psi))=1+3 t+9 t^{2}$. Moreover $h^{0}(\operatorname{Coker}(\psi))=0$. Take a sufficiently large integer $r$ (e.g., $r \geq 6$ ) and a general morphism $\psi^{\prime}: \mathcal{O}(-2)^{\oplus 3} \rightarrow \mathcal{O}^{\oplus r}$, and consider a subbundle morphism $\Psi={ }^{t}\left[\psi^{\prime}, \psi\right]: \mathcal{O}(-2)^{\oplus 3} \rightarrow \mathcal{O}^{\oplus r} \oplus \mathcal{O}(-1)^{\oplus 3}$. Let $\mathcal{E}$ be the cokernel of $\Psi$. Then $\mathcal{E}$ fits in an exact sequence

$$
0 \rightarrow \mathcal{O}^{\oplus r} \stackrel{\varphi}{\rightarrow} \mathcal{E} \rightarrow \operatorname{Coker}(\psi) \rightarrow 0,
$$

where $c_{1}(\mathcal{E})=3$ and $c_{2}(\mathcal{E})=9$. The degeneracy locus $Z$ of the composite of a general inclusion $\mathcal{O}^{\oplus r-1} \hookrightarrow \mathcal{O}^{\oplus r}$ and $\varphi$ has codimension two, and we see that the cokernel of the 
composite is isomorphic to $\mathcal{I}_{Z}(3)$, where $Z$ is a 0 -dimensional closed subscheme of length 9 in $\mathbb{P}^{2}$. Moreover we have the following exact sequence

$$
0 \rightarrow \mathcal{O}_{\mathbb{P}^{2}} \rightarrow \mathcal{I}_{Z}(3) \rightarrow \operatorname{Coker}(\psi) \rightarrow 0 .
$$

Therefore $Z$ lies on the elliptic curve $E$, and $\operatorname{Coker}(\psi)$ is isomorphic to $\mathcal{O}_{E}(\mathfrak{d})$, where $\mathfrak{d}$ is a divisor of degree zero on $E$; thus $\mathcal{E}$ fits in the following exact sequence

$$
0 \rightarrow \mathcal{O}^{\oplus r} \rightarrow \mathcal{E} \rightarrow \mathcal{O}_{E}(\mathfrak{d}) \rightarrow 0 .
$$

This implies that $\mathcal{E}$ is nef. Finally note that $\mathfrak{d} \neq 0$ since $h^{0}(\operatorname{Coker}(\psi))=0$.

We end this section with the following question about some properties of a nef vector bundle $\mathcal{E}$ in case (16) of Theorem 1 .

Question 2. Is the support of the evaluation map $H^{0}(\mathcal{E}) \otimes \mathcal{O} \rightarrow \mathcal{E}$ in case (16) of Theorem 1 necessarily reduced, irreducible and nonsingular?

\section{NeF BUt NON-GLOBALly GENERATED VECTOR BUNDLES}

Lemma 9.1. Let $\mathcal{F}$ be a nef vector bundle on a smooth projective surface $X$. Let $\mathcal{E}_{0}$ be a torsion-free quotient of $\mathcal{F}$, i.e., there exists a surjection $\mathcal{F} \rightarrow \mathcal{E}_{0}$ with $\mathcal{E}_{0}$ a torsion-free coherent sheaf. Let $\mathcal{E}$ denote the double dual $\mathcal{E}_{0}^{\vee \vee}$ of $\mathcal{E}_{0}$. Then $\mathcal{E}$ is a nef vector bundle.

Proof. Since $\mathcal{E}$ is a reflexive sheaf on a smooth surface, $\mathcal{E}$ is a vector bundle. To show that $\mathcal{E}$ is nef, it is enough to show that, for any finite morphism $C \rightarrow X$ from a smooth curve $C$, every quotient line bundle $\mathcal{L}$ of $\left.\mathcal{E}\right|_{C}$ has non-negative degree. Note here that the natural injection $\mathcal{E}_{0} \rightarrow \mathcal{E}$ induces a generically injective morphism $\left.\left.\mathcal{E}_{0}\right|_{C} \rightarrow \mathcal{E}\right|_{C}$. Now let $\mathcal{M}$ be the image of the composite of the morphism $\left.\left.\mathcal{E}_{0}\right|_{C} \rightarrow \mathcal{E}\right|_{C}$ and the surjection $\left.\mathcal{E}\right|_{C} \rightarrow \mathcal{L}$. Since the composite of $\left.\left.\mathcal{F}\right|_{C} \rightarrow \mathcal{E}_{0}\right|_{C}$ and $\left.\mathcal{E}_{0}\right|_{C} \rightarrow \mathcal{M}$ is surjective and $\mathcal{F}$ is nef, we see that $\mathcal{M}$ has non-negative degree. Hence $\mathcal{L}$ has non-negative degree since there is an injection $\mathcal{M} \rightarrow \mathcal{L}$ of line bundles on the smooth curve $C$. Therefore $\mathcal{E}$ is nef.

As is indicated by the statement in Lemma 5.1 (2) (a), we can construct a nef but non-globally generated vector bundle on $\mathbb{P}^{2}$ if $c_{1}=3$ and $c_{2}=8$. See also Example 3 in $\$ 10$ (besides Example 1 in the previous section) for an example of a nef but non-globally generated vector bundle on $\mathbb{P}^{2}$ with $c_{1}=3$ and $c_{2}=9$.

Proof of Proposition Q Given an integer $r \geq 2$ and a closed point $w$ in $\mathbb{P}^{2}$, note first that there exists a section $s$ in $H^{0}\left(\mathcal{O}(3)^{\oplus r+1}\right)$ such that the zero locus $(s)_{0}$ of $s$ is $\{w\}$ as closed subschemes. Let $\varphi: \mathcal{O}(-3) \rightarrow \mathcal{O}^{\oplus r+1}$ be the morphism determined by $s$, and $\mathcal{E}_{0}$ the cokernel of $\varphi$. The dual $\varphi^{\vee}: \mathcal{O}^{\oplus r+1} \rightarrow \mathcal{O}(3)$ of $\varphi$ has $\mathcal{I}_{w}(3)$ as its image, and we obtain an exact sequence

$$
0 \rightarrow \mathcal{E}_{0}^{\vee} \rightarrow \mathcal{O}^{\oplus r+1} \rightarrow \mathcal{I}_{w}(3) \rightarrow 0 .
$$

On the other hand, the ideal sheaf $\mathcal{I}_{w}$ sits in an exact sequence

$$
0 \rightarrow \mathcal{O}(-2) \rightarrow \mathcal{O}(-1)^{\oplus 2} \rightarrow \mathcal{I}_{w} \rightarrow 0 .
$$

Therefore $\operatorname{Tor}_{i}^{\mathcal{O}_{w}}\left(\mathcal{I}_{w}(3), k(w)\right)=0$ for $i>1$. Hence $\mathcal{E}_{0}^{\vee}$ is a vector bundle. The exact sequence (9.1) also implies that $\mathcal{E} x t^{1}\left(\mathcal{I}_{w}(3), \mathcal{O}\right) \cong k(w)$. Let $\mathcal{E}$ be the double dual $\mathcal{E}_{0}^{\vee \vee}$ of $\mathcal{E}_{0}$. Since $\mathcal{H o m}\left(\mathcal{I}_{w}(3), \mathcal{O}\right) \cong \mathcal{O}(-3)$, the vector bundle $\mathcal{E}$ fits in the desired exact sequence

$$
0 \rightarrow \mathcal{O}(-3) \stackrel{\varphi}{\rightarrow} \mathcal{O}^{\oplus r+1} \rightarrow \mathcal{E} \rightarrow k(w) \rightarrow 0 .
$$


Suppose next that a vector bundle $\mathcal{E}$ fits in the exact sequence (9.2). We split the sequence (9.2) into the following two exact sequences:

$$
\begin{gathered}
0 \rightarrow \mathcal{O}(-3) \rightarrow \mathcal{O}^{\oplus r+1} \rightarrow \mathcal{E}_{0} \rightarrow 0 \\
0 \rightarrow \mathcal{E}_{0} \rightarrow \mathcal{E} \rightarrow k(w) \rightarrow 0
\end{gathered}
$$

We see that $\mathcal{E}_{0}$ is a torsion-free sheaf of rank $r$ with $c_{1}\left(\mathcal{E}_{0}\right)=3, c_{2}\left(\mathcal{E}_{0}\right)=9$, and $h^{1}\left(\mathcal{E}_{0}\right)=1$. We have $\mathcal{E} \cong \mathcal{E}_{0}^{\vee \vee}$, and thus $\mathcal{E}$ is a nef vector bundle by Lemma 9.1. Moreover $c_{1}=3$ and $c_{2}=8$. Since $c_{2}<9$, we obtain $h^{1}(\mathcal{E})=0$ by (3.8). Hence $H^{0}\left(\mathcal{E}_{0}\right) \cong H^{0}(\mathcal{E})$. Therefore $\mathcal{E}$ is not globally generated.

Remark 9.1. If $r=2$, the exact sequence in Proposition 20 already appears in [9, 3.2.5]. Professor Adrian Langer kindly informed the author of this fact and that he ruled out this case by mistake.

\section{Some EXAMPles}

Let $X$ be a smooth projective variety, and $\mathcal{E}$ a vector bundle on $X$ of rank $r$. It is well known (see, e.g., [2, Statement(folklore) 4.1]) that if $\mathcal{E}$ is globally generated then $r-1$ general global sections of $\mathcal{E}$ define an injection $\mathcal{O}_{X}^{\oplus r-1} \rightarrow \mathcal{E}$ and this injection extends to an exact sequence

$$
0 \rightarrow \mathcal{O}_{X}^{\oplus r-1} \rightarrow \mathcal{E} \rightarrow \mathcal{I}_{Z} \otimes \operatorname{det} \mathcal{E} \rightarrow 0
$$

where $Z$ is a locally complete intersection subscheme of codimension two in $X$, if not empty. For nef vector bundles, however, analogous results do not hold in general, even if $h^{0}(\mathcal{E}) \geq r-1$, as the following examples show.

Example 2. Let $\mathcal{E}_{0}$ be a nef vector bundle of rank $r-2$ on $\mathbb{P}^{2}$ fitting in the following exact sequence

$$
0 \rightarrow \mathcal{O}(-4) \rightarrow \mathcal{O}^{\oplus r-1} \rightarrow \mathcal{E}_{0} \rightarrow 0 .
$$

Then $r \geq 4$ and $h^{1}\left(\mathcal{E}_{0}\right)=3$. Let $\xi_{1}$ and $\xi_{2}$ be linearly independent elements in $H^{1}\left(\mathcal{E}_{0}\right)$, and let

$$
0 \rightarrow \mathcal{E}_{0} \rightarrow \mathcal{E} \rightarrow \mathcal{O}^{\oplus 2} \rightarrow 0
$$

be the exact sequence whose extension class in $\operatorname{Ext}^{1}\left(\mathcal{O}^{\oplus 2}, \mathcal{E}_{0}\right)$ is determined by $\xi_{1}$ and $\xi_{2}$. Then the connecting homomorphism $H^{0}\left(\mathcal{O}^{\oplus 2}\right) \rightarrow H^{1}\left(\mathcal{E}_{0}\right)$ is injective, and thus $h^{0}(\mathcal{E})=$ $h^{0}\left(\mathcal{E}_{0}\right)=r-1$. Moreover $\mathcal{E}$ is a nef vector bundle of rank $r$ by [10, Theorem 6.2 .12 (ii)]. In this example, every morphism $\mathcal{O}^{\oplus r-1} \rightarrow \mathcal{E}$ is not injective, since it factors through the bundle $\mathcal{E}_{0}$ of rank $r-2$.

Example 3. Let $\mathcal{E}_{0}$ be a nef vector bundle of rank $r-1$ on $\mathbb{P}^{2}$ fitting in the following exact sequence

Then $r \geq 3$ and $h^{1}\left(\mathcal{E}_{0}\right)=1$. Let

$$
0 \rightarrow \mathcal{O}(-3) \rightarrow \mathcal{O}^{\oplus r} \rightarrow \mathcal{E}_{0} \rightarrow 0 .
$$

$$
0 \rightarrow \mathcal{E}_{0} \rightarrow \mathcal{E} \rightarrow \mathcal{O} \rightarrow 0
$$

be a non-split exact sequence; the connecting homomorphism $H^{0}(\mathcal{O}) \rightarrow H^{1}\left(\mathcal{E}_{0}\right)$ is an isomorphism. Then $h^{0}(\mathcal{E})=h^{0}\left(\mathcal{E}_{0}\right)=r$, and it follows from [10, Theorem 6.2.12 (ii)] that $\mathcal{E}$ is a nef but non-globally generated vector bundle of rank $r$ with $c_{1}=3$ and $c_{2}=9$. In this example, a general morphism $\mathcal{O}^{\oplus r-1} \rightarrow \mathcal{E}$ is injective, but its cokernel $\mathcal{C}$ fits in a non-split exact sequence

$$
0 \rightarrow \mathcal{O}_{C} \rightarrow \mathcal{C} \rightarrow \mathcal{O} \rightarrow 0
$$


where $\mathcal{O}_{C}$ is the structure sheaf of some curve $C$ of degree 3 in $\mathbb{P}^{2}$; since $\mathcal{C}$ has a non-zero torsion subsheaf $\mathcal{O}_{C}, \mathcal{C}$ is not isomorphic to a torsion-free coherent sheaf $\mathcal{I}_{Z} \otimes \operatorname{det} \mathcal{E}$ for any closed subscheme $Z$ of $\mathbb{P}^{2}$.

\section{REFERENCES}

[1] Anghel, C., And Manolache, N. Globally generated vector bundles on $\mathbb{P}^{n}$ with $c_{1}=3$. Math. Nachr. 286, 14-15 (2013), 1407-1423.

[2] B̆̆Nică, C. Smooth reflexive sheaves. Rev. Roumaine Math. Pures Appl. 36, 9-10 (1991), 571-593.

[3] Beilinson, A. A. Coherent sheaves on $P^{n}$ and problems in linear algebra. Funktsional. Anal. $i$ Prilozhen. 12, 3 (1978), 214-216.

[4] Bondal, A. I. Representations of associative algebras and coherent sheaves. Izv. Akad. Nauk SSSR Ser. Mat. 53, 1 (1989), 25-44.

[5] Chiodera, L., And Ellia, P. Rank two globally generated vector bundles with $c_{1} \leq 5$. Rend. Istit. Mat. Univ. Trieste 44 (2012), 413-422.

[6] Fulton, W. Intersection theory, second ed., vol. 2 of Ergebnisse der Mathematik und ihrer Grenzgebiete (3). Springer-Verlag, Berlin, 1998.

[7] Kawamata, Y. A generalization of Kodaira-Ramanujam's vanishing theorem. Math. Ann. 261, 1 (1982), 43-46.

[8] Kumar, N. M., Peterson, C., And Rao, A. P. Standard vector bundle deformations on $\mathbb{P}^{n}$. In Vector Bundles and Representation Theory (Columbia, MO, 2002) (Providence, RI, 2003), S. D. Cutkosky, D. Eddidin, Z. Qin, and Q. Zhang, Eds., no. 322 in Contemp. Math., Amer. Math. Soc., pp. 151-163.

[9] LANGER, A. Fano 4-folds with scroll structure. Nagoya Math. J. 150 (1998), 135-176.

[10] Lazarsfeld, R. Positivity in algebraic geometry. II. Positivity for vector bundles, and multiplier ideals., vol. 49 of Ergebnisse der Mathematik und ihrer Grenzgebiete. 3. Folge. A Series of Modern Surveys in Mathematics. Springer-Verlag, Berlin, 2004.

[11] Oнnо, M. Nef vector bundles on a projective space or a hyperquadric with the first Chern class small. arXiv:1409.4191 (2014).

[12] Ohno, M. Nef vector bundles on a projective space with first Chern class 3 and second Chern class 8. Le Matematiche 72, 2 (2017), 69-81.

[13] Ohno, M., ANd Terakawa, H. A spectral sequence and nef vector bundles of the first Chern class two on hyperquadrics. Ann. Univ. Ferrara Sez. VII Sci. Mat. 60, 2 (2014), 397-406.

[14] Okonek, C., Schneider, M., And Spindler, H. Vector bundles on complex projective spaces, vol. 3 of Progress in Mathematics. Birkhäuser, Boston, Mass., 1980.

[15] Peternell, T., Szurek, M., And Wiśniewski, J. A. Numerically effective vector bundles with small Chern classes. In Complex Algebraic varieties, Proceedinigs, Bayreuth, 1990 (Berlin, 1992), T. S. M. Hulek, K. Peternell and F.-O. Schreyer, Eds., no. 1507 in Lecture Notes in Math., Springer, pp. $145-156$.

[16] Sato, E. Uniform vector bundles on a projective space. J. Math. Soc. Japan 28, 1 (1976), 123-132.

[17] Sierra, J. C., And Ugaglia, L. Globally generated vector bundles on projective spaces II. J. Pure Appl. Algebra 218, 1 (2014), 174-180.

[18] Szurek, M., AND Wiśniewski, J. A. On Fano manifolds, which are $\mathbb{P}^{k}$-bundles over $\mathbb{P}^{2}$. Nagoya Math. J. 120 (1990), 89-101.

[19] Tango, H. An example of indecomposable vector bundle of rank $n-1$ on $\mathbf{P}^{n}$. J. Math. Kyoto Univ. 16, 1 (1976), 137-141.

[20] Trautmann, G. Darstellung von Vektorraumbündeln über $\mathbf{C} \backslash\{0\}$. Arch. Math. (Basel) 24 (1973), 303-313.

[21] Vetter, U. Zu einem Satz von G. Trautmann über den Rang gewisser kohärenter analytischer Moduln. Arch. Math. (Basel) 24 (1973), 158-161.

[22] Vienweg, E. Vanishing theorems. J. Reine Angew. Math. 335 (1982), 1-8. 
Graduate School of Informatics and Engineering, The University of Electro-Communications, CHOFU-SHI, TOKYO, 182-8585 JAPAN

E-mail address: masahiro-ohno@uec.ac.jp 